\title{
CHEMISTRY AND KINEMATICS OF THE LATE-FORMING DWARF IRREGULAR GALAXIES LEO A, AQUARIUS, AND SAGITTARIUS DIG
}

\author{
Evan N. Kirby ${ }^{1}$, Luca Rizzi ${ }^{2}$, Enrico V. Held ${ }^{3}$, Judith G. Cohen ${ }^{1}$, Andrew A. ColeE ${ }^{4}$, Ellen M. Manning ${ }^{4}$, \\ Evan D. Skillman ${ }^{5}$, and Daniel R. Weisz ${ }^{6}$ \\ ${ }^{1}$ California Institute of Technology, 1200 E. California Blvd., MC 249-17, Pasadena, CA 91125, USA \\ ${ }^{2}$ W. M. Keck Observatory, 65-1120 Mamalahoa Hwy., Kamuela, HI 96743, USA \\ ${ }^{3}$ INAF, Osservatorio Astronomico di Padova, Vicolo dell'Osservatorio 5, I-35122, Padova, Italy \\ ${ }^{4}$ School of Physical Sciences, University of Tasmania, Private Bag 37, Hobart, Tasmania, 7001 Australia \\ ${ }^{5}$ Minnesota Institute for Astrophysics, University of Minnesota, Minneapolis, MN 55455, USA \\ ${ }^{6}$ Department of Astronomy, 501 Campbell Hall, University of California, Berkeley, CA 94720, USA \\ Received 2016 September 27; revised 2016 October 24; accepted 2016 October 26; published 2016 December 22
}

\begin{abstract}
We present Keck/DEIMOS spectroscopy of individual stars in the relatively isolated Local Group dwarf galaxies Leo A, Aquarius, and the Sagittarius dwarf irregular galaxy. The three galaxies-but especially Leo A and Aquarius-share in common delayed star formation histories (SFHs) relative to many other isolated dwarf galaxies. The stars in all three galaxies are supported by dispersion. We found no evidence of stellar velocity structure, even for Aquarius, which has rotating $\mathrm{H}$ I gas. The velocity dispersions indicate that all three galaxies are dark-matter-dominated, with dark-to-baryonic mass ratios ranging from $4.4_{-0.8}^{+1.0}$ (SagDIG) to 9.6 $6_{-1.8}^{+2.5}$ (Aquarius). Leo A and SagDIG have lower stellar metallicities than Aquarius, and they also have higher gas fractions, both of which would be expected if Aquarius were further along in its chemical evolution. The metallicity distribution of Leo A is inconsistent with a closed or leaky box model of chemical evolution, suggesting that the galaxy was preenriched or acquired external gas during star formation. The metallicities of stars increased steadily for all three galaxies, but possibly at different rates. The $[\alpha / \mathrm{Fe}]$ ratios at a given $[\mathrm{Fe} / \mathrm{H}]$ are lower than that of the Sculptor dwarf spheroidal galaxy, which indicates more extended SFHs than Sculptor, consistent with photometrically derived SFHs. Overall, the bulk kinematic and chemical properties for the late-forming dwarf galaxies do not diverge significantly from those of less delayed dwarf galaxies, including dwarf spheroidal galaxies.
\end{abstract}

Key words: galaxies: abundances - galaxies: dwarf - galaxies: individual (Leo A, DDO 210, Sgr dIG) Local Group - stars: abundances

Supporting material: machine-readable tables

\section{INTRODUCTION}

The Local Group plays host to dozens of dwarf galaxies. These galaxies are laboratories for star formation and chemical evolution because they span a huge range of age, stellar mass, metallicity, morphology, and gas fraction. A glance at Hubble Space Telescope (HST) color-magnitude diagrams of Local Group dwarf galaxies reveals an expansive diversity of stellar populations (Holtzman et al. 2006; Weisz et al. 2014). For example, deep HST imaging of the dwarf spheroidal galaxy (dSph) Cetus shows no evidence of young stars (Monelli et al. 2010b), whereas similarly deep HST imaging of the dwarf irregular galaxy (dIrr) Aquarius shows old red giants, an intermediate-age main-sequence turnoff (MSTO), and young stars on the main sequence (Cole et al. 2014). Both Cetus and Aquarius are isolated and have stellar masses of about $2 \times 10^{6} M_{\odot}$, yet their star formation histories (SFHs) are very different.

It is clear that environment plays a big role in shaping the SFHs and the gas content of these galaxies (e.g., van den Bergh 1994). For example, no dwarf galaxy within $\sim 300 \mathrm{kpc}$ of the Milky Way (MW) except the Magellanic Clouds has any gas (Grcevich \& Putman 2009; Spekkens et al. 2014).

* The data presented herein were obtained at the W. M. Keck Observatory, which is operated as a scientific partnership among the California Institute of Technology, the University of California, and the National Aeronautics and Space Administration. The Observatory was made possible by the generous financial support of the W. M. Keck Foundation.
Furthermore, all of these satellite galaxies are old, spheroidal, and supported by dispersion rather than rotation. On the other hand, with only three exceptions (Cetus, Tucana, and Eridanus II; Monelli et al. 2010a, 2010b; Li et al. 2016), all of the Local Group dwarf galaxies farther than $300 \mathrm{kpc}$ have gas and at least some young stars. They also tend to be characterized by irregularly distributed young stellar populations, and some of them rotate. The environment effect persists far outside the Local Group. Nearly all galaxies with $M_{*}>10^{7} M_{\odot}$ farther than $1.5 \mathrm{Mpc}$ from a massive host galaxy are currently forming stars, but proximity to a host can terminate star formation (Geha et al. 2012). However, it is worth noting that present isolation does not ensure that a galaxy was always isolated. For example, one possible reason for Cetus's and Tucana's lack of star formation is that they were once close enough to the MW or M31 to experience ram pressure and/or tidal stripping (Teyssier et al. 2012).

While it is clear that many dwarf galaxies lack young populations, every dwarf galaxy contains at least some ancient stars older than 10 Gyr (Mateo 1998; Orban et al. 2008; Weisz et al. 2014). Both isolated and satellite galaxies can have intermediate-aged stellar populations. Examples include the satellite dSphs Fornax and Leo I and the relatively isolated dIrrs IC 1613 and NGC 6822. Apparently all dwarf galaxies start with at least a skeleton population of ancient stars. Some galaxies manage to build later populations, whereas othersalmost entirely satellites of larger hosts-do not. 
However, the stellar chemical properties of the dwarf galaxies do not depend on environment to nearly the same degree as the gas content. All dwarf galaxies obey the same relationship between stellar mass and stellar metallicity, regardless of environment or SFH (Skillman et al. 1989a; Kirby et al. 2013). Therefore, metallicity and chemical evolution seem to be inextricably tied to the stellar mass of a galaxy. Although environment influences gas fraction and $\mathrm{SFH}$, it does not overtly influence chemistry.

The galaxies with the best-constrained SFHs are those with space-based photometry that reaches at least as deep as the MSTO of the oldest stellar population. Such data are especially resource intensive for the isolated galaxies, which are farther away than MW satellite galaxies. The Local Cosmology from Isolated Dwarfs (LCID; Bernard et al. 2008, 2009) project has collected HST imaging of isolated dwarfs. Skillman et al. (2014) compared the SFHs of the six LCID dwarfs: the dSphs Cetus and Tucana, the dIrrs IC 1613 and Leo A, and the transition dwarfs LGS 3 and Phoenix. To date, the only other isolated galaxies with comparable data quality are Leo T (Weisz et al. 2012) and Aquarius (Cole et al. 2014).

With this deep HST imaging, Cole et al. (2007, 2014) found that Leo A (DDO 69) and Aquarius (DDO 210) both experienced highly delayed star formation relative to other dIrrs. Like all dwarf galaxies, Leo A and Aquarius have ancient populations. However, they distinguish themselves by having formed less than $20 \%$ of their stars more than 6-8 Gyr ago. They are also notable for being among the most isolated galaxies in the local Galactic neighborhood. Both galaxies are probably members of the Local Group. Although they lie right at the zero-velocity surface that separates the Local Group from the Hubble flow (Karachentsev et al. 2009), their velocities relative to the Local Group barycenter suggest that they are bound to the group (McConnachie 2012). However, their free-fall times to the MW, M31, or the Local Group barycenter are on the order of a Hubble time, which means that they have been living and will continue to live in the most remote regions of the Local Group essentially forever.

The isolation and delayed SFHs of Leo A and Aquarius led us to conduct a spectroscopic survey of their red giants. We also included the Sagittarius dIrr (SagDIG) in this study because it is also as isolated as the other two dIrrs. The gas and stellar populations of all three dIrrs have low probabilities $(\leqslant 2 \%)$ of ever having been influenced by the MW (Teyssier et al. 2012). SagDIG's SFH is not as extreme as that of Leo A or Aquarius (Weisz et al. 2014). Instead, about $40 \%$ of its stars are ancient. However, like Leo A and Aquarius, it then stopped forming stars for several gigayears before restarting. It is worth keeping in mind that the interpretation of SagDIG's SFH is based on shallower HST imaging that does not reach the ancient MSTO. We now discuss some other interesting properties of each galaxy to keep in mind when interpreting the spectroscopic data.

\subsection{Leo A}

Using HST/WFPC2 photometry, Tolstoy et al. (1998) first noticed that Leo A conspicuously lacked a prominent ancient ( $>10$ Gyr) population. For that reason, it stood out among the Local Group and challenged the idea that all dwarf galaxies are old. The claim of the absence of an old population was not without controversy. Also using HST/WFPC2 photometry, Schulte-Ladbeck et al. (2002) claimed that Leo A was predominantly ancient, and Dolphin et al. (2002, 2003) confirmed the presence of a small number of RR Lyrae, which are necessarily ancient, as well as an old, metal-poor halo around the galaxy. It was not until Cole et al. (2007) obtained HST imaging down to the old MSTO that the fraction of ancient stars was conclusively determined to be less than $10 \%$.

The SFH that Cole et al. derived shows that most of the star formation happened more recently than $6 \mathrm{Gyr}$ ago, and it ramped up from then until $2 \mathrm{Gyr}$ ago. The present star formation rate (SFR) is measured as $9.3 \times 10^{-5} M_{\odot} \mathrm{yr}^{-1}$ from $\mathrm{H} \alpha$ imaging and $6.0 \times 10^{-4} M_{\odot} \mathrm{yr}^{-1}$ from ultraviolet (UV) imaging (Karachentsev \& Kaisina 2013). The discrepancy for dwarf galaxies is well known (Lee et al. 2009), but the UV rate is generally regarded as more robust. At the present UVmeasured SFR, Leo A could have formed all of its stars in $5.5 \mathrm{Gyr}$, which is broadly consistent with the late SFH observed by Cole et al. (2007).

The best constraints from broadband photometry have not detected long-term trends in the average stellar metallicity, which appears to be constant at $[\mathrm{Fe} / \mathrm{H}]=-1.4$ (Cole et al. 2007). A peculiarly low $\mathrm{H}$ II region metallicity $([\mathrm{O} / \mathrm{H}]=$ $-1.31 \pm 0.10$; van Zee et al. 2006) and consequently low effective yield (Lee et al. 2006) also make Leo A stand out among dIrrs. The previously measured average stellar metallicity $(\langle[\mathrm{Fe} / \mathrm{H}]\rangle=-1.58$; Kirby et al. 2013) is not far below the gas-phase metallicity. The potential similarity in metallicity between stars and gas possibly corroborates the slow evolution in metallicity observed by Cole et al. (2007). In other words, the present (gas-phase) metallicity has not progressed much beyond the past-averaged (stellar) metallicity.

Early HI maps hinted that Leo A is not rotationally supported (Allsopp 1978). Higher-resolution maps showed some rotation of the gas, but the rotation is not aligned with the stellar disk (Young \& Lo 1996). A large, low-velocity feature near the center of the galaxy-near the bulk of the stars-could be seen as the receding half of a nearly edge-on disk, but Young \& Lo suggested that it is a contracting or expanding shell, possibly due to a recent supernova. Instead of rotation, they measured a mostly dispersion-supported galaxy with $\sigma_{v}=$ $9 \mathrm{~km} \mathrm{~s}^{-1}$. This dispersion could have been consistent with no dark matter if the HI gas is supported by its own internal pressure. However, Brown et al. (2007) measured the stellar velocity dispersion from $10 \mathrm{~B}$ supergiants. They also obtained $\sigma_{v}=9 \mathrm{~km} \mathrm{~s}^{-1}$. Later, Kirby et al. (2014) measured $\sigma_{v}=6.4_{-1.2}^{+1.4} \mathrm{~km} \mathrm{~s}^{-1}$ from red giants. Either measurement of the stellar velocity dispersion conclusively indicates that the galaxy is supported by the dynamical pressure provided by a dark matter subhalo rather than hydrodynamical pressure.

\subsection{Aquarius}

Other than the HST-based SFH of Cole et al. (2014), Aquarius is the least well studied of the three galaxies presented here. A few surveys have examined the carbon stars (Gullieuszik et al. 2007) and variable stars (Ordoñez \& Sarajedini 2016) in Aquarius. The latter study found both RR Lyrae and Cepheids, indicating the presence of ancient ( $>10 \mathrm{Gyr})$ and fairly young ( $300 \mathrm{Myr})$ populations. Interestingly, the young Cepheids were offset from the galactic center, where the older Cepheids were found. Ordoñez \& Sarajedini interpreted this displacement as stellar migration, though the stars could have formed in an off-center star formation region. 
Although Cole et al. (2014) found that star formation in Aquarius experienced a late resurgence, $\mathrm{H} \alpha$ and UV imaging show that its current SFR is zero (van Zee 2000; Hunter et al. 2010). In contrast to that of Leo A, the SFR of Aquarius seems to have tapered off recently. It is not clear whether the current lack of star formation is permanent or a temporary lull, possibly as the result of recent stellar feedback. The presence of copious H I gas (Lo et al. 1993) suggests that the galaxy is not yet finished with star formation.

\section{3. $\mathrm{Sag} D I G$}

SagDIG has the highest gas fraction $\left(M(\mathrm{HI}) / M_{*}=\right.$ $4.6 \pm 1.2$ ) of any galaxy in the Local Group. The high gas fraction also imparts a high specific SFR to SagDIG. The UVmeasured SFR is $7.2 \times 10^{-4} M_{\odot} \mathrm{yr}^{-1}$ (Karachentsev \& Kaisina 2013), corresponding to a specific SFR of $0.4 \mathrm{Gyr}^{-1}$. That makes SagDIG one of the fastest-growing galaxies in the Local Group. It could have grown to its present stellar mass at its present SFR in just 2.5 Gyr.

SagDIG's high specific SFR makes its metallicity very interesting. It has a very low gas-phase metallicity $([\mathrm{O} / \mathrm{H}]=$ -1.4; Skillman et al. 1989b; Saviane et al. 2002). That ties SagDIG with Leo A for the distinction of being the most oxygenpoor galaxy in the Local Group, with an oxygen abundance nearly as low as the quintessentially metal-poor galaxy I Zw 18 (Searle \& Sargent 1972; Skillman \& Kennicutt 1993).

Ground-based, wide-field imaging shows that the stellar population of SagDIG is elongated and very extended. The surface brightness profile drops exponentially out to $5^{\prime}$, at which point it fades into the background (Beccari et al. 2014). The galaxy may even be embedded in a very low density stellar halo (Higgs et al. 2016). Curiously, the stellar distribution seems to have little to do with the H I distribution. Whereas the stellar distribution is smooth and elongated, the neutral gas is round. It is also asymmetric, with an apparent hole just to the southwest of the galaxy's center.

A closed box model of chemical evolution coupled with the measured gas fraction (revised for possible gas loss) and gasphase metallicity suggests that the average stellar metallicity of SagDIG should be $[\mathrm{Fe} / \mathrm{H}]=-2$ (Saviane et al. 2002). This value is exactly in accord with photometric estimates of the stellar metallicity (Momany et al. 2002, 2005). However, it is $2.3 \sigma$ lower than the stellar metallicity predicted by the spectroscopically measured stellar mass-stellar metallicity relation (Kirby et al. 2013). A spectroscopic measurement of the stellar metallicity would answer whether the closed box model and the photometric estimate of the metallicity are valid or whether SagDIG conforms to the mass-metallicity relation.

\section{SPECTROSCOPIC OBSERVATIONS}

Kirby et al. (2014) already published some Keck/DEIMOS (Faber et al. 2003) spectroscopy of stars in Leo A and Aquarius. We obtained additional DEIMOS spectra of individual stars in those galaxies, as well as SagDIG. This section describes those observations.

\subsection{Source Catalogs}

We designed DEIMOS slit masks using photometry and astrometry from multiple sources. For Leo A, we used the $V$ and I Subaru/Suprime-Cam (Miyazaki et al. 2002) catalog of Stonkutè et al. (2014). For Aquarius, we used the catalog of
McConnachie et al. (2006), who observed the galaxy with Suprime-Cam in the $V$ and $I$ filters. For SagDIG, we used two different photometry catalogs. We combined Momany et al.'s (2002) photometry from the ESO Multimode Instrument (Dekker et al. 1986) at the ESO New Technology Telescope (NTT) with Momany et al.'s (2014) photometry from the HST/ Advanced Camera for Surveys (ACS). Although the ACS photometry is more accurate than the NTT photometry, the field of view of ACS is only $3 ! 4$ square. Hence, we supplemented the ACS photometry with the NTT photometry, which spans a 6!2 square. The NTT photometry was obtained in $V$ and $I$ filters, and we converted the ACS F475W, F606W, and $\mathrm{F} 814 \mathrm{~W}$ magnitudes into $V$ and $I$ with the transformation equations of Saha et al. (2011). All magnitudes were corrected for extinction with the Schlegel et al. (1998) dust maps.

\subsection{Target Selection}

DEIMOS slit mask design constraints required that we prioritize targets for placement on the slit masks. We first prioritized stars that were likely to be members of the red giant branch (RGB) of their respective galaxy. We identified the RGB by overlaying theoretical isochrones on the colormagnitude diagram (CMD). We used Yonsei-Yale isochrones in the $V$ and $I$ filters (Demarque et al. 2004). The isochrones were shifted to the distance modulus appropriate for each galaxy: $(m-\mathrm{M})_{0}=24.59$ for Leo A (Dolphin et al. 2002; Tammann et al. 2011), 24.95 for Aquarius (Cole et al. 2014), and 25.10 for SagDIG (Momany et al. 2005).

We chose two isochrones to bound the red and blue sides of the RGB. The red side had an age of $14 \mathrm{Gyr}$ and a metallicity of $[\mathrm{Fe} / \mathrm{H}]=0$. The blue side had an age of $2 \mathrm{Gyr}$ and the minimum metallicity of the isochrone set: $[\mathrm{Fe} / \mathrm{H}]=-3.8$ and -2.2 for Yonsei-Yale and Padova, respectively. Stars near the middle of the color range of these two bounding isochrones were given higher priority. Brighter stars were also given higher priority. Finally, we selected stars outside of the red and blue bounding isochrones to fill any remaining space on the slit mask. Extra targets limit the lengths of the longest slits, which made for easier data reduction because the focal plane curvature is not noticeable for short slits.

In principle, any selection in the CMD could impose a bias in the age and metallicity distributions derived for the stars. For example, if the bounding isochrone on the red is too stringent, the selection will exclude metal-rich and/or old stars. In practice, our selection box is generous. The color selection likely does not impose any significant bias in the age and metallicity distributions except to exclude very young stars that have not reached or will never reach the RGB. However, our sample may have a slight metallicity bias due to the magnitude limit. The magnitudes of stars near the top of the RGB are especially sensitive to metallicity. Metal-poor stars are brighter. Therefore, some of the most metal-rich stars in our sample will be fainter and have spectra with lower signal-to-noise ratio (S/ $\mathrm{N})$ than metal-poor stars at the same evolutionary phase, leading to slight bias against metal-rich stars. The effect would not bias our results beyond the error bars that we quote.

Figures 1-3 show the CMDs and coordinate maps of the galaxies. Stars that we identified to be members of their respective galaxies (Section 3.3) are shown as blue filled circles. Stars that failed any of the membership criteria are shown as red crosses. Orange plus signs show stars whose spectra were so noisy that we could not measure a radial 

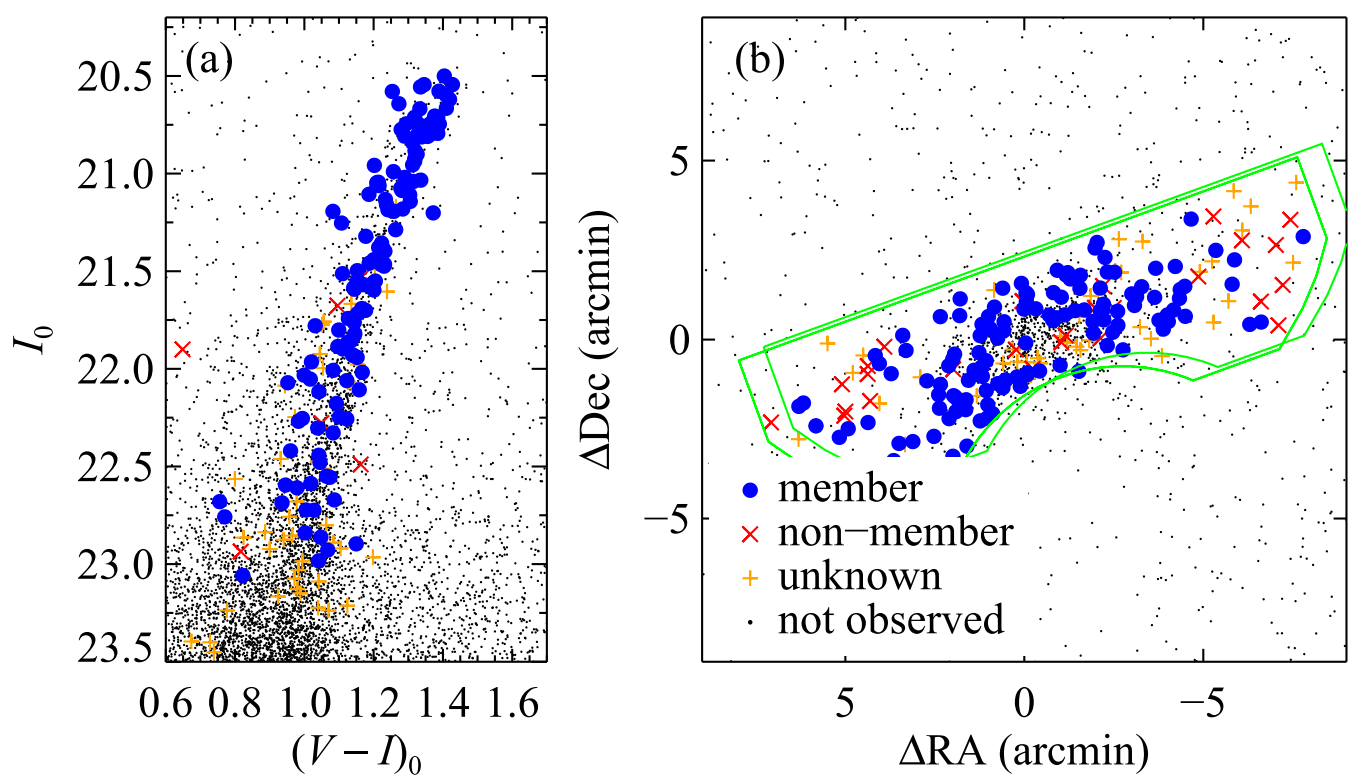

Figure 1. (a) CMD of Leo A from Stonkute et al.'s (2014) photometry catalog. Stars confirmed to be members are shown as blue filled circles, nonmembers are shown as red crosses, stars of inconclusive membership are shown as orange plus signs, and objects not observed with DEIMOS are shown as black filled circles. (b) The sky in the area of the DEIMOS observations. The outlines of the DEIMOS slit masks are shown in green.

velocity. Small black filled circles identify stars for which we did not obtain a spectrum.

\subsection{Observations}

We observed the three galaxies with DEIMOS over several nights in 2013 and 2014. We used the 1200G grating, which has a groove spacing of $1200 \mathrm{~mm}^{-1}$ and a blaze wavelength of $7760 \AA$. We set the central wavelength to $7800 \AA$, and we used the OG550 order-blocking filter. This configuration provides a resolving power of $R \sim 7000$ at the central wavelength. We obtained images of an internal quartz lamp for flat-fielding and internal $\mathrm{Ne}, \mathrm{Ar}, \mathrm{Kr}$, and $\mathrm{Xe}$ arc lamps for wavelength calibration. Table 1 lists the dates and conditions of the observations. This paper includes two slit masks, leoaaW and aqra, that were previously published by Kirby et al. (2013).

We reduced the data with the spec2d pipeline (Cooper et al. 2012; Newman et al. 2013). The pipeline extracts rectangular areas of the $\mathrm{CCD}$ image corresponding to the spectrally dispersed images of the slit. Each slit image is flatfielded and wavelength-calibrated. The wavelength calibration is based on the arc lamp images and refined by the measured pixel positions of terrestrial sky emission lines. The stellar spectrum is automatically identified and extracted with optimal weighting. We incorporated some modifications to spec2d that improve the wavelength calibration and account for the curvature of the 2D spectrum due to differential atmospheric refraction (Kirby et al. 2015b, 2015c). The pipeline tracks the variance of the spectrum so that the final 1D spectrum has both flux and an error on the flux.

The slit masks sagdia, sagdib, and aqra had observations separated by more than 1 month. For these slit masks, we grouped together the observations taken in the same week and reduced them together. That left us with two independent sets of reduced 1D spectra from the same slit mask. We applied a heliocentric correction to the wavelength array of the second reduction to bring it into the same velocity reference frame as the first reduction. Then, we co-added the two sets of spectra, weighting each pixel by its inverse variance. Furthermore, some stars were observed on multiple slit masks. We co-added their spectra in the same manner in order to achieve the best $\mathrm{S} / \mathrm{N}$ for each star.

Figure 4 shows example spectra in each of the galaxies. The spectra were chosen to illustrate the full range of $\mathrm{S} / \mathrm{N}$ in our data set. The topmost spectrum, LeoA 29587, shows an example that was co-added from two different slit masks (leoaaW and leoac).

\section{SPECTROSCOPIC MEASUREMENTS}

We measured radial velocities and metallicities from the DEIMOS spectra of individual stars in the three dIrrs. We used the radial velocities to help determine whether each targeted star was a member of the galaxy.

\subsection{Radial Velocities}

Our technique to measure the radial velocities (based on Simon \& Geha 2007) is the same as we have used before. The technique relies on matching the observed spectrum to empirical templates. This process is similar to the DEEP2 collaboration's galaxy redshift measurement technique (Newman et al. 2013). Specifically, the velocity $v_{\mathrm{obs}}$ is measured by minimizing $\chi^{2}$ between the observed spectrum and a template spectrum. The template with the lowest $\chi^{2}$ is the one used for the final velocity measurement. We used the nine metal-poor stars observed as radial velocity standards by Kirby et al. (2015c). We transformed all velocities into the heliocentric frame.

The observed velocity of the star can vary with the position of the star perpendicular to the slit. Ideally, the star will be centered in the slit, but misalignment can translate into a spurious velocity offset of a few kilometers per second with respect to the arc lines or sky emission lines, which fill the slit. The offset can be corrected by establishing a geocentric frame of reference based on the telluric absorption lines (Simon \& Geha 2007; Sohn et al. 2007). However, some of our spectra span observations separated by months. The heliocentric velocity zero point shifts significantly over that time. As a 

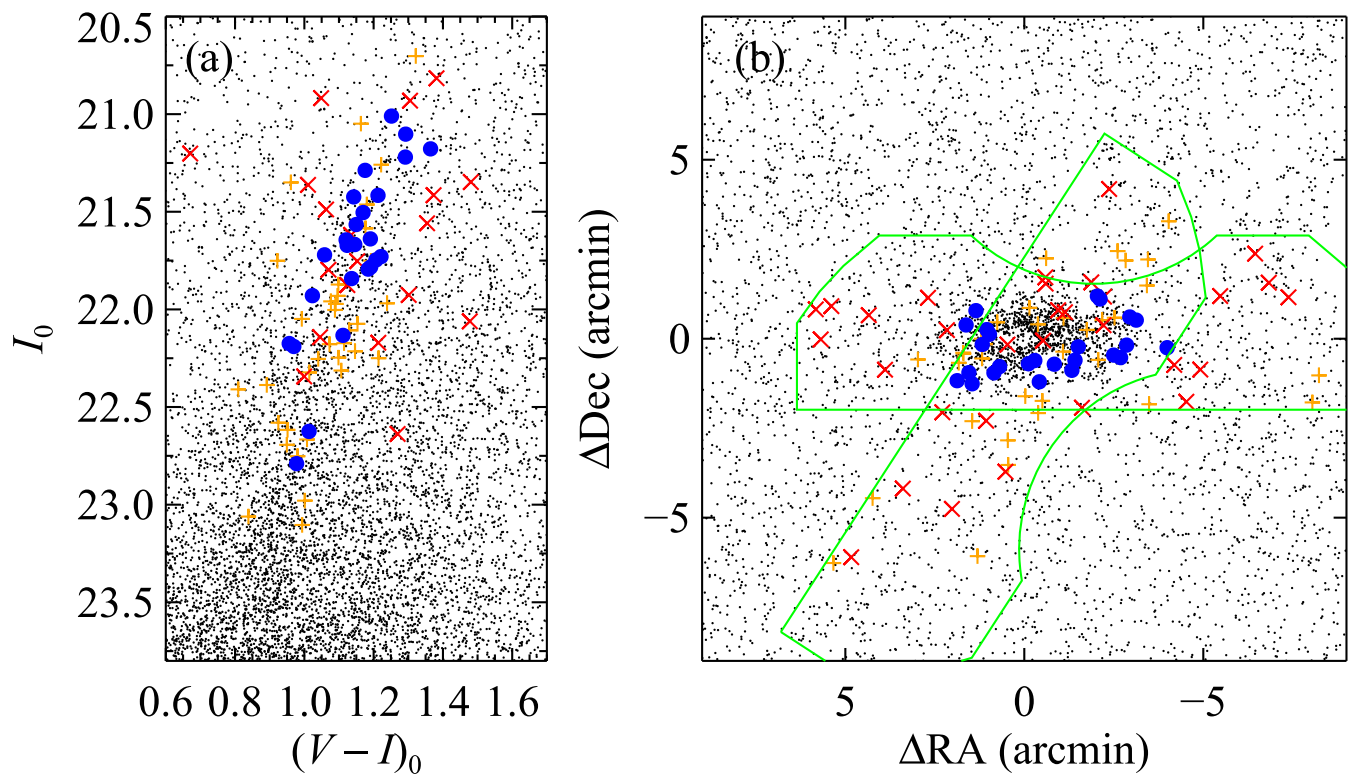

Figure 2. Same as Figure 1, but for Aquarius. The photometry comes from McConnachie et al. (2006).

result, the telluric lines in the stacked spectra are smeared over a range of wavelengths, precluding a clean measurement of the slit mis-centering correction. Consequently, we did not perform this correction on any of our data. Regardless, the correction is much less important for dIrrs than ultrafaint dSphs (e.g., Simon \& Geha 2007; Kirby et al. 2015a, 2015c) because the typical magnitude of the correction is $2.5 \mathrm{~km} \mathrm{~s}^{-1}$, much smaller than the velocity dispersions of dIrrs. Furthermore, the correction becomes more important for observations where the seeing drops below the slit width. That was not the case for most of our observations.

We estimated random velocity uncertainty by resampling the observed spectrum 1000 times. In each Monte Carlo trial, we added noise in the spectrum. The added noise in each pixel was sampled from a Gaussian random distribution with a width equal to the square root of the variance on that pixel's flux. Hence, each pixel was perturbed on average by $1 \sigma$ from its nominal value. We remeasured the velocity from the noiseadded spectrum, considering only the radial velocity template that best matched the original spectrum. The random uncertainty, $\delta_{\text {rand }} v$, is the standard deviation of $v_{\text {helio }}$ measured from all 1000 trials. Simon \& Geha (2007) found that this estimate of velocity uncertainty was too small for spectra with high S/Ns. They calculated a systematic error, $\delta_{\text {sys }} v$, based on repeat measurements of the same stars. Kirby et al. (2015c) also followed that procedure to determine $\delta_{\text {sys }} v=1.49 \mathrm{~km} \mathrm{~s}^{-1}$. The final error on each measurement is $\delta v=\sqrt{\delta_{\text {sys }} v^{2}+\delta_{\text {sys }} v^{2}}$.

\subsection{Chemical Abundances}

We measured metallicities and some detailed abundance ratios by spectral synthesis. We used the same technique as Kirby et al. (2008, 2010), who give more details than provided here. Specifically, we compared the observed spectra with a large grid of synthetic spectra spanning the expected ranges for old red giants of effective temperature, surface gravity, metallicity, and $\alpha$-element enhancement.

We prepared the spectra for abundance measurements first by dividing by the spectrum of a hot star to correct for telluric absorption. Then, we fit a polynomial to line-free regions of the spectrum. This polynomial was the first attempt at determining the stellar continuum. We then used Levenberg-Marquardt minimization to search the grid of synthetic spectra for the model spectrum with the minimum $\chi^{2}$ when compared with the observed, continuum-normalized spectrum. We fixed the surface gravity at the value determined by fitting model isochrones to the star's broadband magnitude and color. The temperature, $[\mathrm{Fe} / \mathrm{H}]$, and $[\alpha / \mathrm{Fe}]$ were free parameters, although the temperature was constrained by both the spectrum and the broadband photometry. After determining the best-fit atmospheric parameters, we fit a new continuum polynomial to the quotient of the observed spectrum and the best-fitting model. We continually refit the spectrum with successive refinements of the continuum until the procedure converged. Finally, we measured individual abundances of $\mathrm{Mg}, \mathrm{Si}, \mathrm{Ca}$, and $\mathrm{Ti}^{7}$ by restricting the spectral fit to regions of the spectrum that contain absorption lines of each of those elements.

We estimated random uncertainties on the abundances from the diagonal elements of the covariance matrix produced by the Levenberg-Marquardt grid search. Like the radial velocity uncertainties, the random uncertainty is an incomplete estimate of the error. We computed the total error on an element's abundance by adding a systematic error in quadrature with the random error. Kirby et al. (2010) determined the magnitude of the systematic errors by comparing repeated measurements of the same stars. The systematic errors are 0.106 dex for $[\mathrm{Fe} / \mathrm{H}]$, $0.065 \mathrm{dex}$ for $[\mathrm{Mg} / \mathrm{Fe}], 0.113 \mathrm{dex}$ for $[\mathrm{Si} / \mathrm{Fe}], 0.111 \mathrm{dex}$ for $[\mathrm{Ca} / \mathrm{Fe}]$, and $0.090 \mathrm{dex}$ for $[\mathrm{Ti} / \mathrm{Fe}]$.

\subsection{Membership}

Not all stars that we observed belong to the dIrr galaxies that we are studying. We required every star to pass three membership criteria: (1) position in the CMD, (2) absence of spectral features that would indicate nonmembership, and (3) radial velocity.

\footnotetext{
7 We adopted solar abundances of $\epsilon(\mathrm{Fe}) \equiv 12+\log [n(\mathrm{Fe}) / n(\mathrm{H})]=7.52$ (Sneden et al. 1992), $\epsilon(\mathrm{Mg})=7.58, \epsilon(\mathrm{Si})=7.55, \epsilon(\mathrm{Ca})=6.36$, and $\epsilon(\mathrm{Ti})=$ 4.99 (Anders \& Grevesse 1989).
} 

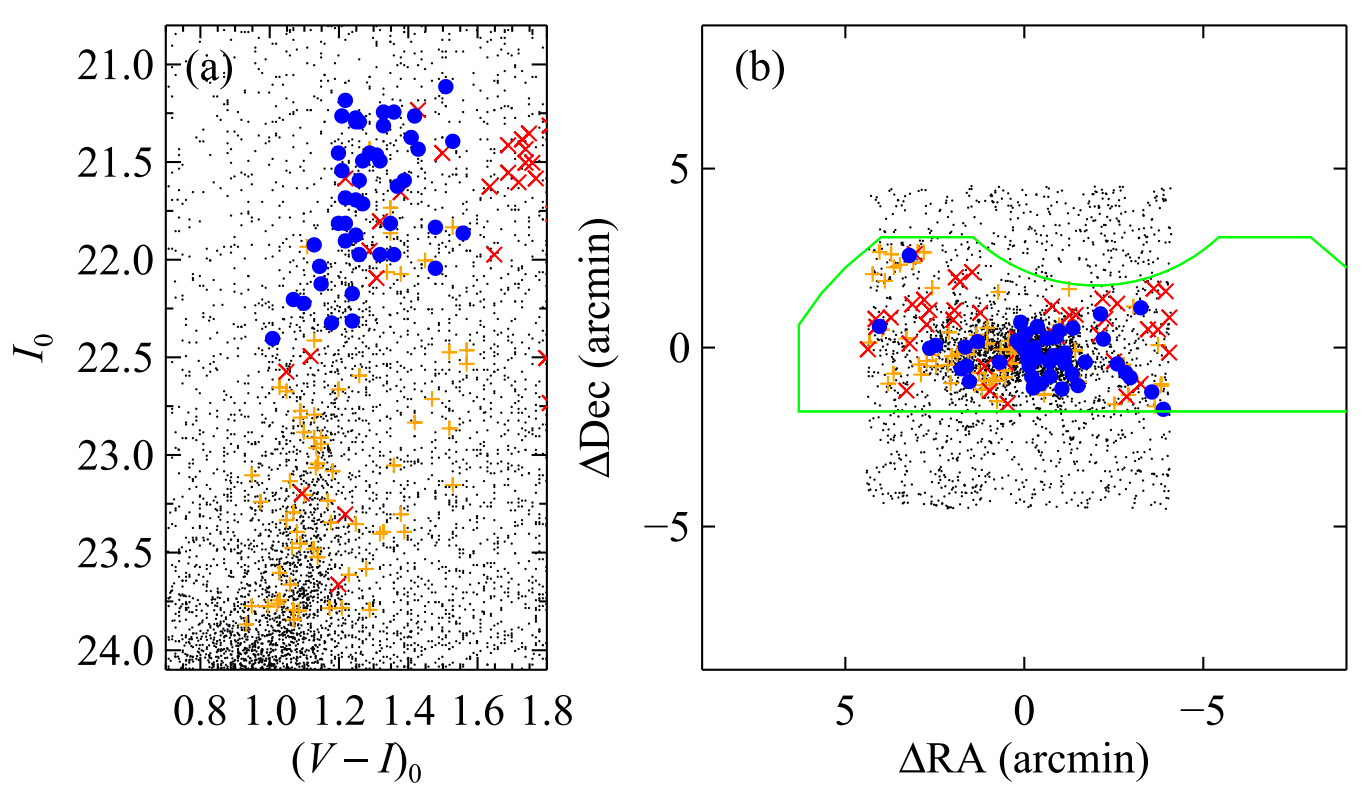

Figure 3. Same as Figure 1, but for SagDIG. The photometry comes from Momany et al. (2002, 2014).

First, the stars need to have the approximate colors and magnitudes of red giants at the distance of each galaxy. Although we prioritized the design of the DEIMOS slit masks to target such stars, we also targeted some stars that could not be red giant members based on their position in the CMD. Any star that did not fall within the bounds of the extreme red and blue isochrones described in Section 2.2 was excluded.

Second, we examined each spectrum for spectral features that indicate that the star could not be a red giant member of the galaxy. The most obvious such features were redshifted emission lines, such as $\mathrm{H} \alpha$ or $[\mathrm{O} I I] \lambda 3727$. These features indicated that the target was a background galaxy. Another telltale spectral feature was the Na I $\lambda 8190$ doublet. This feature is very strong in dwarf stars. At the distances of dIrrs, dwarf stars would be much fainter than the apparent magnitudes of our target stars. Hence, a strong Na doublet indicates that the star is in the foreground (Spinrad \& Taylor 1971; Cohen et al. 1978). We measured equivalent widths by fitting Gaussians for weaker doublets and Lorentzians for stronger doublets. Kirby et al. (2012) found that a combined equivalent width of the two Na lines stronger than $1 \AA$ indicates that the star is a dwarf for any reasonable range of $\mathrm{Na}$ abundance. We adopted the same membership criterion.

We also noted the presence of $\mathrm{TiO}$ and $\mathrm{CN}$ bands. TiO is generally found in cool, metal-rich stars. Hence, the presence of $\mathrm{TiO}$ is a likely indicator of nonmembership, but it is not definitive. Hence, we did not use it to determine membership. Regardless, every star with visible $\mathrm{TiO}$ bands failed at least one other membership criterion. $\mathrm{CN}$ bands usually indicate that a star is enhanced in carbon. Carbon stars on the RGB or asymptotic giant branch (AGB) are not particularly rare, even in metal-poor dwarf galaxies. Hence, we did not use the presence of $\mathrm{CN}$ as a membership indicator. However, we did exclude any stars with obvious $\mathrm{CN}$ from the measurement of chemical abundances because $\mathrm{CN}$ lines are not adequately represented in our spectral syntheses.

Third, we identified member stars on the basis of their radial velocities. We followed the same process as our previous work on the kinematics of dwarf galaxies (Kirby et al. 2014, 2015c). We required that member stars have radial velocities that fall within $2.58 \sigma_{v}$ of the mean velocity, $\left\langle v_{\text {helio }}\right\rangle$, where $\sigma_{v}$ is the velocity dispersion (Section 4). This criterion includes $99 \%$ of the member stars if their velocities are normally distributed. However, some of the stars have velocity errors on the order of $\sigma_{v}$. To accommodate these stars, we also count as members all stars whose $1 \sigma$ velocity uncertainty overlaps the $\pm 2.58 \sigma_{v}$ velocity range. Put concisely, the velocity requirement for membership is $\left|v_{\text {helio }}-\left\langle v_{\text {helio }}\right\rangle\right|-\delta v<2.58 \sigma_{v}$.

Figure 5 shows the velocity distributions of stars near the mean velocity for each dIrr. The range of the plots includes all member stars but not all nonmembers. Black shading indicates nonmembers that were excluded because of their CMD position or the presence of a strong $\mathrm{Na}$ doublet. Red shading indicates stars that were excluded only on the basis of radial velocity. Background galaxies are not shown.

Table 2 gives the names, coordinates, extinction-corrected $I_{0}$ magnitude, reddening-corrected $(V-I)_{0}$ color, number of slit masks on which the star was observed, S/N, radial velocity, and membership (yes or no). The last column indicates various reasons for nonmembership or other qualities of the spectrum, such as the presence of $\mathrm{CN}$ absorption in member stars. The table contains 197 member stars and 123 nonmembers for all three dIrrs. Table 3 gives temperatures, gravities, and abundances for the 179 stars where those values were measurable. The last column, $[\alpha / \mathrm{Fe}]$, is not an arithmetic average of the previous four columns. Rather, it is a determination of the $[\alpha / \mathrm{Fe}]$ ratio using all of the available $\alpha$ element lines in the spectrum. In this sense, it is an average weighted by the $\mathrm{S} / \mathrm{N}$ of the relevant $\mathrm{Mg}, \mathrm{Si}, \mathrm{Ca}$, and $\mathrm{Ti}$ absorption lines. This is the value called $[\alpha / \mathrm{Fe}]_{\mathrm{atm}}$ by Kirby et al. (2010).

We compared our membership list for SagDIG with Momany et al.'s (2014) proper-motion-culled catalog. The field of view of $H S T$, from which the proper motions were measured, limits the overlap between that catalog and our DEIMOS catalog to 30 stars. Of them, we classified 22 as members, all of which have proper motions less than

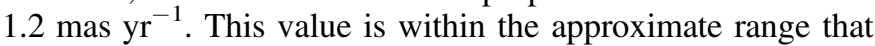
Momany et al. considered stars to pass the proper-motion cut. Of the eight stars that we classified as nonmembers, only one 
Table 1

DEIMOS Observations

\begin{tabular}{|c|c|c|c|c|c|c|c|c|}
\hline Galaxy & Slit Mask & Targets & $\begin{array}{l}\text { Slit Width } \\
(\operatorname{arcsec})\end{array}$ & $\begin{array}{l}\text { Tot. Exp. Time } \\
\text { (hr) }\end{array}$ & $\begin{array}{l}\text { Exp. Time } \\
\text { (minutes) }\end{array}$ & Exposures & $\begin{array}{l}\text { Seeing } \\
(\operatorname{arcsec})\end{array}$ & UT Date \\
\hline \multirow[t]{2}{*}{$\overline{\text { Leo A }}$} & leoaaW ${ }^{\mathrm{a}}$ & 121 & 1.1 & 6.7 & 400 & 14 & 0.9 & 2013 Jan 14 \\
\hline & leoA & 75 & 0.7 & 4.0 & 240 & 12 & 1.0 & 2013 Apr 1 \\
\hline \multirow[t]{3}{*}{ Aquarius } & aqra $^{a}$ & 77 & 0.7 & 8.9 & 333 & 12 & 0.5 & 2013 Jul 8 \\
\hline & & & & & 60 & 2 & 0.7 & 2013 Sep 1 \\
\hline & & & & & 142 & 5 & 0.9 & 2013 Sep 2 \\
\hline & sagdia & & & & 240 & 8 & 0.7 & 2014 Aug 28 \\
\hline & \multirow[t]{4}{*}{ sagdib } & 85 & 0.7 & 11.0 & 330 & 11 & 0.8 & 2014 Jun 30 \\
\hline & & & & & 30 & 1 & 0.9 & 2014 Aug 29 \\
\hline & & & & & 150 & 5 & 0.9 & 2014 Aug 30 \\
\hline & & & & & 150 & 5 & 0.6 & 2014 Aug 31 \\
\hline
\end{tabular}

Note.

${ }^{\text {a }}$ Observations published by Kirby et al. (2013).

had a proper motion in excess of 1.2 mas $\mathrm{yr}^{-1}$. Thus, the proper-motion cut does not misclassify members as nonmembers, but it is not especially efficient at ruling out nonmembers.

\section{KINEMATICS}

\subsection{Velocity Dispersion}

We measured the velocity dispersions of each dIrr using maximum likelihood. Our approach is similar to that of Walker et al. (2006) and identical to that of Kirby et al. (2015c). The likelihood, $L$, that the galaxy has a mean velocity $\left\langle v_{\text {helio }}\right\rangle$ and a velocity dispersion $\sigma_{v}$ is

$$
\begin{aligned}
\ln L= & -\frac{1}{2} \sum_{i}^{N} \ln \left[2 \pi\left((\delta v)_{i}^{2}+\sigma_{v}^{2}\right)\right] \\
& -\frac{1}{2} \sum_{i}^{N}\left(\frac{\left(\left(v_{\text {helio }}\right)_{i}-\left\langle v_{\text {helio }}\right\rangle\right)^{2}}{(\delta v)_{i}^{2}+\sigma_{v}^{2}}\right) .
\end{aligned}
$$

The sum is performed over the velocity measurements of the $N$ unique member stars in each dIrr. The velocity and error on the $i$ th star are $\left(v_{\text {helio }}\right)_{i}$ and $(\delta v)_{i}$ (see Section 3.1). We maximized the likelihood using a Markov chain Monte Carlo (MCMC) method with a Metropolis-Hastings algorithm.

The determination of $\left\langle v_{\text {helio }}\right\rangle$ and $\sigma_{v}$ depends on the stars included in the member list. However, the velocity criterion for membership (Section 3.3) depends on $\left\langle v_{\text {helio }}\right\rangle$ and $\sigma_{v}$. Therefore, these two parameters must be determined iteratively. First, we made rough guesses at the mean velocity and velocity dispersion by calculating the mean and standard deviation of possible members in the velocity ranges shown in Figure 5. These values were used to construct the initial member list. Second, we reevaluated $\left\langle v_{\text {helio }}\right\rangle$ and $\sigma_{v}$ using Equation (1) and an MCMC chain with $10^{5}$ iterations. These values were used to refine the member list. Third, we repeated this process until the membership list remained the same from one iteration to the next. Finally, we reevaluated $\left\langle v_{\text {helio }}\right\rangle$ and $\sigma_{v}$ with the final member list using an MCMC chain with $10^{7}$ iterations. The difference between $10^{5}$ and $10^{7}$ iterations is not significant enough to alter the member list, but it does help us better determine the confidence intervals on the measurements.

Using a Markov chain allows us to sample the parameter space well enough to determine one-sided confidence intervals.
We quote error bars as the values that enclose $68.3 \%$ of the MCMC trials. The error bars are allowed to be asymmetric, such that the parameter space between the lower error bar and the mean includes $34.2 \%$ of the trials, and the parameter space between the mean and the upper error bar includes another $34.2 \%$ of the trials.

The dynamical mass of a galaxy can be estimated from its line-of-sight velocity dispersion and projected half-light radius. Although there is some uncertainty from the unknown velocity anisotropy and from the deprojection of the 2D half-light radius to its 3D value, Wolf et al. (2010) found a robust mass estimator that minimizes the effect of these uncertainties. The mass within the $3 \mathrm{D}$ half-light radius is $M_{1 / 2}=4 G^{-1} \sigma_{v}^{2} r_{h}$, where $\sigma_{v}$ is the line-of-sight velocity dispersion and $r_{h}$ is the $2 \mathrm{D}$ half-light radius. These two quantities are directly measured from observations.

Table 4 gives our measurements and confidence intervals for $\left\langle v_{\text {helio }}\right\rangle, \sigma_{v}$, and $M_{1 / 2}$. All of the galaxies have velocity dispersions in excess of what would be expected from stellar mass alone. The $V$-band mass-to-light ratios of the known matter (stars and $\mathrm{H}$ I gas) range from 1.6 to $2.2 M_{\odot} L_{\odot}^{-1}$, but the observed dynamical mass-to-light ratios range from 10 to $21 M_{\odot} L_{\odot}^{-1}$. Thus, dark matter outweighs luminous matter by at least a factor of four. This finding is consistent with the velocity dispersions of H I gas (e.g., Lo et al. 1993; Young \& Lo 1996). However, the stars are not subject to hydrodynamical pressure. Thus, we can conclusively rule out gas dynamics as the origin of the velocity dispersion. Instead, the galaxies must be dark matter dominated.

\subsection{Rotation}

The tidal stirring model (Mayer et al. 2001) posits that rotationally supported dIrrs transform into dispersion-supported $\mathrm{dSphs}$ in the tidal field of a massive host galaxy. However, Wheeler et al. (2015) found that stellar rotation is not common among dIrrs. The lack of rotation lessens the need for tides to transform a dIrr's dynamical support from rotation to dispersion. Wheeler et al. already established that Leo A and Aquarius have limited rotation based on Kirby et al.'s (2014) data. However, the limit on Aquarius was not particularly stringent due to the limited $\mathrm{S} / \mathrm{N}$ of the spectra. In this section, we test for stellar rotation in our current spectroscopic samples. 


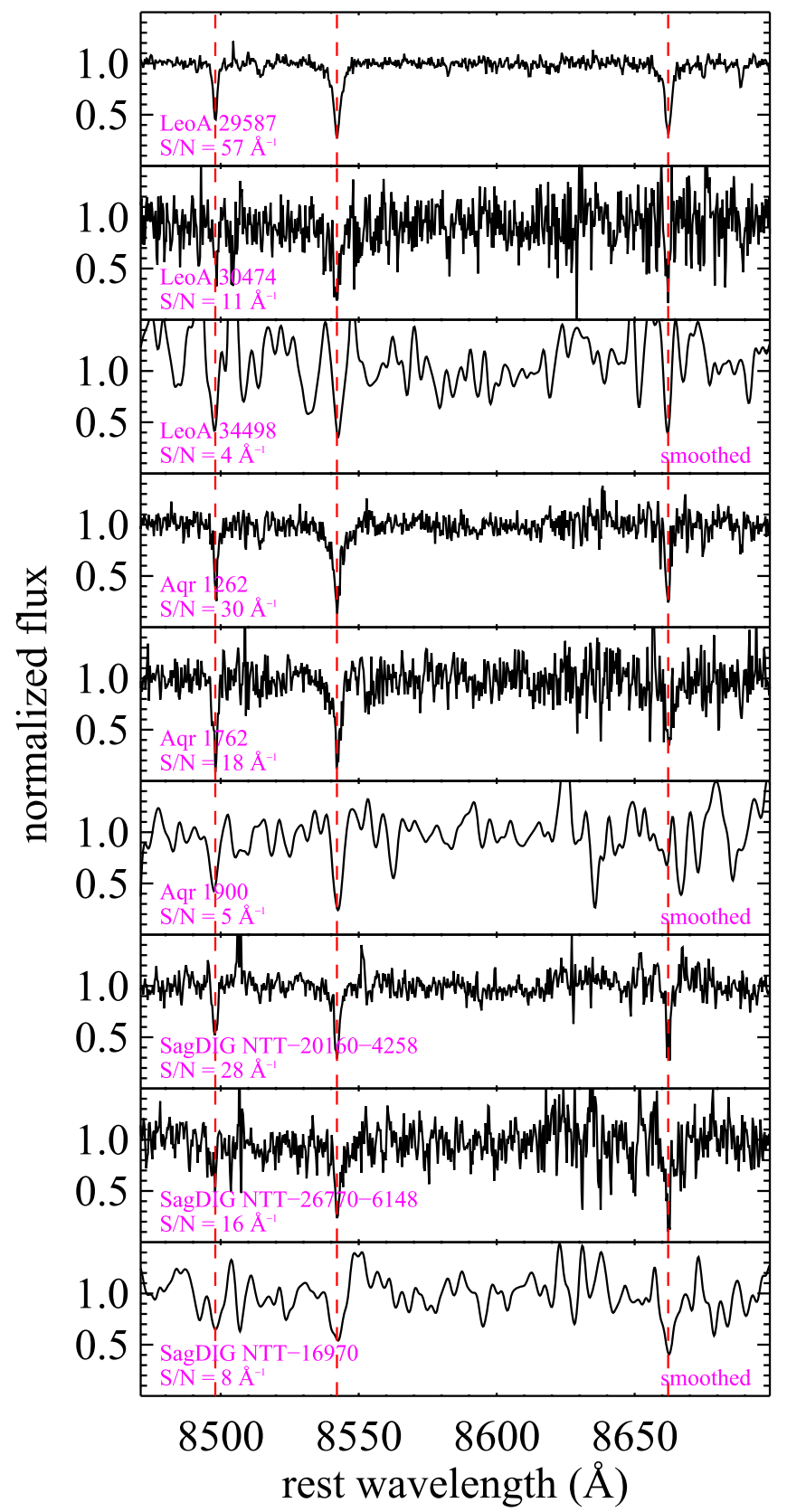

Figure 4. DEIMOS spectra with the highest, median, and lowest $\mathrm{S} / \mathrm{N}$ of the member stars for each of Leo A, Aquarius, and SagDIG. Red dashed lines indicate the rest wavelengths of the Ca II triplet. For display only, the spectra with the lowest $\mathrm{S} / \mathrm{N}$ are smoothed to reduce noise. Each panel gives the name of each star and the $\mathrm{S} / \mathrm{N}$ of the spectrum.

The most straightforward way to test for rotation is to plot the stars' velocities versus their distances from the minor axis. If the galaxy is a rotationally supported disk, then the average velocity on one side of the minor axis should be blueshifted relative to the mean velocity, and the velocity on the other side should be redshifted. The left panels of Figure 6 show this diagnostic. No rotation is apparent. The average velocities on either side of the minor axis seem about the same. Given that some galaxies have been found to exhibit prolate rotation (e.g., Andromeda II; Ho et al. 2012) — that is, rotation around the isophotal major axisFigure 6 also shows the velocities versus their distances from the major axis. This type of rotation is not common, and indeed, we do not observe it in any of the three dIrrs.
We also tested for rotation in a statistically rigorous manner. Following Wheeler et al. (2015), we modeled the velocity of each star with a rotation term: $\left\langle v_{\text {helio }}\right\rangle+v_{\text {rot }} \cos \left(\theta-\theta_{i}\right)$. The magnitude of rotation is $v_{\text {rot }}$, and the axis of rotation is defined by the position angle $\theta$. The position angle of each star is $\theta_{i}$. This parameterization allows the rotation to occur around any axis, not just the minor axis. We found the maximum likelihood values for $v_{\text {rot }}, \theta,\left\langle v_{\text {helio }}\right\rangle$, and $\sigma_{v}$. For computational efficiency, we maximized the logarithm of the likelihood:

$$
\begin{aligned}
\ln L= & -\frac{1}{2} \sum_{i}^{N} \ln \left[2 \pi\left((\delta v)_{i}^{2}+\sigma_{v}^{2}\right)\right]-\frac{1}{2} \\
& \times \sum_{i}^{N}\left(\frac{\left[\left(v_{\text {helio }}\right)_{i}-\left(\left\langle v_{\text {helio }}\right\rangle+v_{\text {rot }} \cos \left(\theta-\theta_{i}\right)\right)\right]^{2}}{(\delta v)_{i}^{2}+\sigma_{v}^{2}}\right) .
\end{aligned}
$$

Equation (2) is a generalization of Equation (1). In order to determine $v_{\text {rot }}$ and $\theta$ freely and without bias, we did not constrain $\left\langle v_{\text {helio }}\right\rangle$ or $\sigma_{v}$ to match the values we previously determined. Regardless, the final values computed from Equation (2) were indistinguishable from those computed from Equation (1). As before, we maximized the likelihood with an MCMC chain with $10^{5}$ links.

We did not detect rotation in the dIrrs. We constrained the rotation to be $v_{\text {rot }}<5.0,9.0$, and $6.0 \mathrm{~km} \mathrm{~s}^{-1}$ with $95 \%$ confidence in Leo A, Aquarius, and SagDIG, respectively. The corresponding limits on the ratio of rotation velocity to velocity dispersion are $v_{\text {rot }} / \sigma_{v}<0.6,1.1$, and 0.6. This result is consistent with the results of Wheeler et al. (2015). Thus, we have tightened the constraints on rotation in Leo $\mathrm{A}$ and Aquarius and added SagDIG to the list of nonrotating dIrrs.

\subsection{Comparison of the Stellar and Gas Kinematics}

Local Group dIrrs are the nearest gas-rich galaxies. As such, they have been observed fairly extensively with $21 \mathrm{~cm}$ radio measurements. All three of Leo A, Aquarius, and SagDIG contain copious amounts of gas with gas-to-stellar mass ratios of $2.3 \pm 0.5,1.4 \pm 0.2$, and $4.6 \pm 1.2$ (Hunter et al. 2012). It is interesting to compare the kinematics of the gas with the kinematics of the stars. The gas traces the recent history of the galaxy, as well as hydrodynamical effects. On the other hand, the stars retain a longer dynamical memory than the gas, and they are unaffected by collisional pressure.

We compared our measurements of stellar velocities with observations of the $\mathrm{HI}$ gas by Local Irregulars that Trace Luminosity Extremes: The H I Nearby Galaxy Survey (LITTLE THINGS; Hunter et al. 2012). The $21 \mathrm{~cm}$ observations come from the Very Large Array (VLA). Figure 7 shows the robustweighted galaxy maps with velocity color coding for both the stars and gas. The figure also shows the H I surface density contours.

For each galaxy, we computed the mean, flux-weighted velocity from the robust-weighted VLA maps: $\left\langle v_{\text {helio }}\right\rangle(\mathrm{HI})=$ $\sum f(\mathrm{H}) v(\mathrm{H}) / \sum f(\mathrm{H})$, where $f(\mathrm{H})$ is the H I surface density in a pixel and $v(\mathrm{H})$ is the velocity from the intensity-weighted, firstmoment map. We computed a similar flux-weighted velocity dispersion: $\sigma_{v}(\mathrm{H} \mathrm{I})=\sum f(\mathrm{H}) \sigma_{v}(\mathrm{H}) / \sum f(\mathrm{H})$. In this case, $\sigma_{v}(\mathrm{H})$ comes from the intensity-weighted, second-moment map. Table 4 shows those values, which can be compared to the stellar velocities in the same table. In general, the mean gas velocities agree with the mean stellar velocities. The gas 

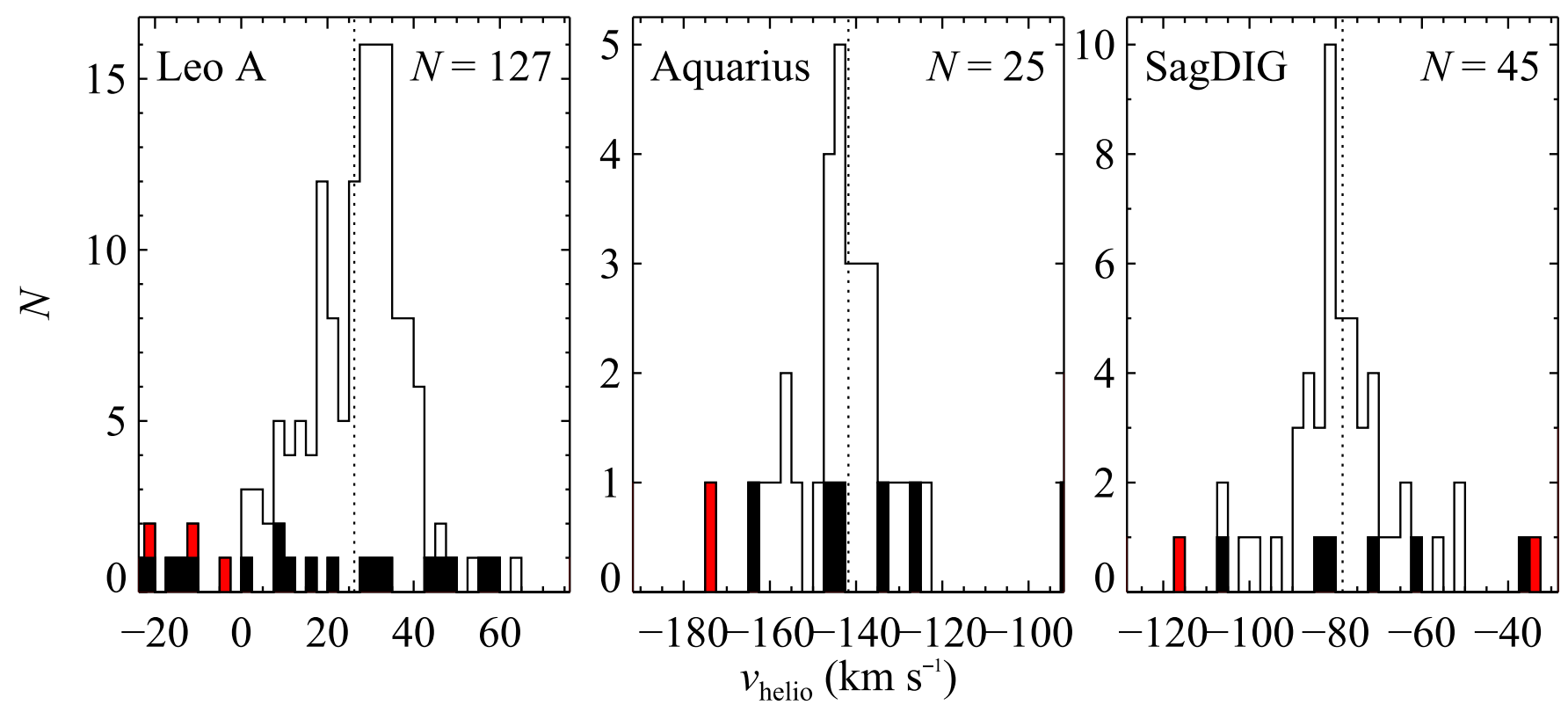

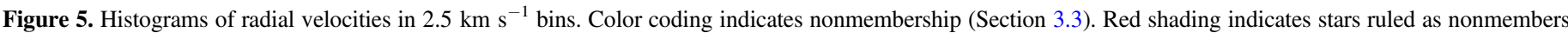

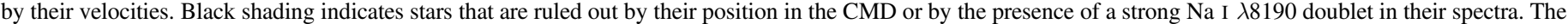
vertical dotted line indicates the mean radial velocity. The upper right corners show the number of member stars.

velocity dispersions are slightly less than the stellar velocity dispersions. However, the gas velocities explicitly exclude rotation. Low levels of stellar rotation or even stellar binaries could slightly inflate the stellar velocity dispersions.

Leo A, like all the dIrrs presented here, shows no sign of stellar rotation or any velocity structure. On the other hand, the $\mathrm{HI}$ velocity structure is more complex. Although there is no obvious rotation, there is some structure in the surface density and the velocity field. The majority of gas appears in an arc just north of the galaxy's center. The two sides of the arc appear as two lobes of gas. The velocity varies along the arc, but not in a way that indicates rotation. A blob of gas just east of Leo A's center is blueshifted relative to the mean velocity. Furthermore, there is an area just southwest of the center that is devoid of gas. Although it appears from Figure 7 that this area is also devoid of stars, it is coincidental that we did not obtain spectra there. Optical imaging shows that stars do fill the H I hole. The contrast between structure in the gas and lack thereof in the stars emphasizes that the gas traces the recent dynamical evolution of the galaxy, whereas the stars have been relaxed over many dynamical times.

Aquarius is the only one of the three dIrrs to show rotation in the gas. The gas rotates about a north-south axis, with a velocity differential of about $10 \mathrm{~km} \mathrm{~s}^{-1}$ from east to west. Interestingly, the stellar distribution is elongated in the same direction, as if the stars formed a disk that rotates in the same plane as the gas. However, the stellar velocities show no evidence for rotation. Therefore, the fact that the stellar isophotes and the gas rotation share the same axis is either coincidental or reflective of the underlying structure of dark matter. For example, the dark matter halo may be triaxial, with a long axis pointing east-west. This is merely a speculative suggestion in the absence of many more stellar velocity measurements that could constrain the velocity anisotropy. Additionally, spectra of the younger blue stars would reveal to what degree the old RGB stars are representative of the entire stellar population.
SagDIG has an H I hole, like Leo A. It is possible that a recent supernova explosion carved the hole in the gas. The explosion would not have affected the positions of the stars, which fill the hole. However, Momany et al. (2014) found that even young stars can be found in the hole, which could indicate that the hole was formed by gravitational instability rather than stellar feedback. On the other hand, a study of similar kiloparsec-sized H I holes in five nearby dIrrs found that the underlying stellar population produced sufficient energy to create the holes (Warren et al. 2011). Ignoring the hole, the gas density contours are fairly circular. If the gas is rotating in a disk, then we are viewing the disk face-on. As a result, we would not be able to detect any rotation along the line of sight.

\subsection{Comparison to Other Dwarf Galaxies}

Figure 8 shows some dynamical properties of the three dIrrs with other Local Group galaxies. Velocity dispersion, dynamical mass, and mass-to-light ratio within the half-light radius are shown as a function of stellar mass. These quantities follow well-defined trends with stellar mass. Velocity dispersion and mass increase and mass-to-light ratio decreases with mass. Leo A, Aquarius, and SagDIG fall well within the ranges of these quantities defined by other Local Group galaxies.

The color coding in the plot distinguishes MW satellites from isolated galaxies. The three dIrrs in this paper fall in the latter group. Regardless, the two groups do not show different trends. The lack of dichotomy in $\sigma_{v}$ and $M_{1 / 2}$ is not surprising. The environmental influence of the MW is not expected to drastically affect the dark matter density in the center of the galaxy (the region that dictates the stellar velocities) until just before the galaxy is completely disrupted by tides (Peñarrubia et al. 2008). Environment could potentially affect $M / L_{V}$ because ram pressure stripping truncates star formation. The sudden cessation of stellar mass growth might be expected to stunt galaxy growth, resulting in a lower luminosity for a given mass. Stripping also causes satellite galaxies to be older on average than field galaxies, which would also reduce $L_{V}$ at a 
Table 2

Radial Velocities and Membership

\begin{tabular}{|c|c|c|c|c|c|c|c|c|c|c|}
\hline Galaxy & ID & R.A. (J2000) & Decl. (J2000) & $\begin{array}{c}I_{0} \\
(\mathrm{mag})\end{array}$ & $\begin{array}{c}(V-I)_{0} \\
(\mathrm{mag})\end{array}$ & Masks & $\begin{array}{l}\mathrm{S} / \mathrm{N}^{\mathrm{a}} \\
\left(\mathrm{A}^{-1}\right)\end{array}$ & $\begin{array}{c}v_{\text {helio }} \\
\left(\mathrm{km} \mathrm{s}^{-1}\right)\end{array}$ & Member? & Flags $^{\mathrm{b}}$ \\
\hline LeoA & 29115 & 095903.87 & +304632.6 & 18.64 & 2.29 & 1 & 97 & $0.1 \pm 1.5$ & $\mathrm{~N}$ & $\mathrm{TiO}$ v CMD Na \\
\hline LeoA & 31326 & 095904.81 & +304808.9 & 21.89 & 1.10 & 3 & 15 & $32.2 \pm 3.1$ & $\mathrm{Y}$ & $\cdots$ \\
\hline LeoA & 9282 & 095905.57 & +304525.9 & 21.51 & 1.11 & 1 & 6 & $35.0 \pm 5.6$ & $\mathrm{Y}$ & $\mathrm{CN}$ \\
\hline LeoA & 11200 & 095905.64 & +304616.2 & 21.60 & 1.20 & 1 & 7 & $37.7 \pm 5.1$ & $\mathrm{Y}$ & $\cdots$ \\
\hline LeoA & 11058 & 095906.22 & +304610.9 & 21.70 & 1.18 & 1 & 6 & $38.5 \pm 7.9$ & $\mathrm{Y}$ & $\cdots$ \\
\hline LeoA & 30011 & 095906.33 & +304556.0 & 21.02 & 1.29 & 2 & 31 & $16.7 \pm 2.0$ & $\mathrm{Y}$ & $\cdots$ \\
\hline LeoA & 34130 & 095906.87 & +304649.5 & 22.90 & 1.15 & 2 & 6 & $37.1 \pm 34.7$ & $\mathrm{Y}$ & $\cdots$ \\
\hline LeoA & 31582 & 095907.09 & +304536.5 & 22.27 & 0.98 & 2 & 7 & $28.3 \pm 6.1$ & $\mathrm{Y}$ & $\ldots$ \\
\hline LeoA & 31580 & 095907.78 & +304517.2 & 22.05 & 1.02 & 2 & 12 & $11.5 \pm 3.5$ & $\mathrm{Y}$ & $\cdots$ \\
\hline LeoA & 9397 & 095907.90 & +304528.2 & 21.11 & 1.30 & 1 & 12 & $17.8 \pm 3.2$ & $\mathrm{Y}$ & $\ldots$ \\
\hline
\end{tabular}

Notes.

To convert to $\mathrm{S} / \mathrm{N}$ per pixel, multiply by 0.57 .

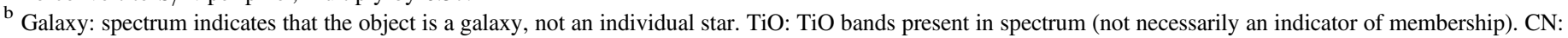

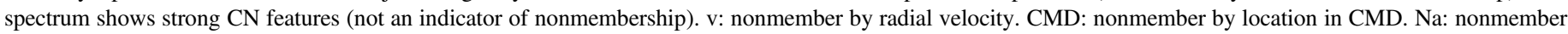
by presence of strong $\mathrm{Na}$ I $\lambda 8190$ doublet.

(This table is available in its entirety in machine-readable form.)

given stellar mass. Nonetheless, the satellite galaxies do not show a higher $M / L_{V}$ on average than the isolated galaxies. The effect of environment on this quantity seems to be below our ability to detect it.

\section{CHEMICAL COMPOSITION}

The metallicity distributions of dwarf galaxies record the details of the history of gas flow in the galaxies while stars were forming. For example, in a "closed box" model of chemical evolution (Schmidt 1963; Talbot \& Arnett 1971), the mean stellar metallicity approaches the stellar yield as the gas supply runs out. The model can be modified to a "leaky box" by allowing gas to escape. If the gas escapes at a rate proportional to the SFR (outflow $=\eta \times \mathrm{SFR}$ ), then the yield, $p$, can simply be replaced by the effective yield: $p_{\text {eff }}=p /$ $(1+\eta)$. In the limit where the true yield is invariant among galaxies, the average metallicity can be used as a proxy for the amount of gas lost during the lifetime of star formation.

Panel (d) of Figure 8 shows the relation between the average stellar metallicities, $\langle[\mathrm{Fe} / \mathrm{H}]\rangle$, and stellar mass for dwarf galaxies. Leo A, Aquarius, and SagDIG fit in the trend defined by other dwarf galaxies (Kirby et al. 2011b, 2013). The relation is linear for dwarf galaxies $\left(10^{4}<M_{*} / M_{\odot}<10^{8}\right)$. Larger galaxies continue this trend up to about $10^{9} M_{\odot}$, and even larger galaxies are continuous with the smaller galaxies, though the slope flattens above $10^{9} M_{\odot}$ (Gallazzi et al. 2005). Interpreted in the context of the leaky box model, the larger galaxies have deeper gravitational potential wells, which allows them to retain more gas (smaller $\eta$ ) in the face of stellar feedback.

As Kirby et al. (2013) pointed out, dIrrs are indistinguishable from $\mathrm{dSphs}$ on the mass-metallicity relation. Despite still possessing gas and forming stars, the average metallicity depends much more strongly on stellar mass than on the amount of star formation the galaxy has yet to complete. However, the presence of gas allows an analysis not possible for the dSphs: comparison between gas-phase and stellar metallicities. ${ }^{8}$ For example, Leo A and SagDIG, as very low

\footnotetext{
8 The gas-phase metallicity in Aquarius is not measurable because it has no current star formation and therefore no $\mathrm{H}$ II regions from which gas-phase metallicity could be measured (van Zee et al. 1997).
}

luminosity dIrrs, have low metallicities appropriate for their low stellar mass (Berg et al. 2012). It turns out that they also lie low on the stellar mass-stellar metallicity relation.

Their low metallicities could be a consequence of their high gas fractions. In the closed box model, the gas fraction decreases over time, which establishes a monotonic, inverse proportionality between gas fraction and metallicity. Pagel (1997) presented the relations between present metallicity, yield, gas fraction, and average stellar metallicity. The yield is $p=Z /\left(\ln \mu^{-1}\right)$ (Pagel's Equation (8.6)), where $Z$ is the current gas-phase metallicity and $\mu$ is the gas fraction. The gas fractions for Leo A and SagDIG are 0.69 and 0.82, respectively (see Table 4). The gas-phase metallicities are $4.9 \%$ (van Zee et al. 2006) and 4.0\% (Saviane et al. 2002) of the solar value. The yields, then, are 0.13 and $0.20 Z_{\odot}$. The average stellar metallicity is $p(1+\mu \ln \mu /(1-\mu))$ (Pagel's Equation (8.8)), corresponding to $[\mathrm{M} / \mathrm{H}]=-1.6$ and -1.7 for Leo $\mathrm{A}$ and SagDIG, respectively. These values are strikingly close to our measurements of $\langle[\mathrm{Fe} / \mathrm{H}]\rangle=-1.67_{-0.08}^{+0.09}$ and $-1.88_{-0.09}^{+0.13}$.

The preceding argument is very rough. First, the conclusions depend on how well the galaxy approximates a closed box. A variable gas-loss rate could heavily affect the determination of the yield. For example, Saviane et al. (2002) estimated that gas loss from SagDIG would lead to a larger value for the "true" gas fraction. Their prediction for the stellar metallicity $([\mathrm{M} / \mathrm{H}]$ $=-2$ ) ended up being slightly too low, but they were right to point out that gas loss needs to be considered in a more careful model of chemical evolution. Second, the gas-phase metallicities are most sensitive to oxygen, whereas the stellar metallicities are based on iron absorption lines. Because the $\mathrm{O} / \mathrm{Fe}$ ratio is unknown, we implicitly assumed that the value is solar. In reality, the value will depend on the changing ratio of Type II and Type Ia supernova ejecta in the galaxy's chemical evolution.

\subsection{Metallicity Distributions}

While the average metallicity of a galaxy does contain a lot of information, resolved stellar spectroscopy affords an even more valuable diagnostic of chemical evolution. Specifically, the shape of the metallicity distribution depends on the details 
Table 3

Chemical Abundances

\begin{tabular}{|c|c|c|c|c|c|c|c|c|c|}
\hline Dwarf & ID & $\begin{array}{l}T_{\text {eff }} \\
(\mathrm{K})\end{array}$ & $\log _{\left(\mathrm{cm} \mathrm{s}^{-2}\right)}$ & {$[\mathrm{Fe} / \mathrm{H}]$} & {$[\mathrm{Mg} / \mathrm{Fe}]$} & {$[\mathrm{Si} / \mathrm{Fe}]$} & {$[\mathrm{Ca} / \mathrm{Fe}]$} & {$[\mathrm{Ti} / \mathrm{Fe}]$} & {$[\alpha / \mathrm{Fe}]$} \\
\hline LeoA & 33186 & 4584 & 1.54 & $-1.82 \pm 0.30$ & $\cdots$ & & & & \\
\hline LeoA & 29857 & 4299 & 0.63 & $-2.51 \pm 0.12$ & $\ldots$ & $+0.85 \pm 0.21$ & $+0.21 \pm 0.29$ & $+0.73 \pm 0.25$ & $+0.09 \pm 0.33$ \\
\hline LeoA & 30073 & 4295 & 0.76 & $-2.02 \pm 0.11$ & $\ldots$ & $+0.47 \pm 0.21$ & $-0.03 \pm 0.25$ & $+0.35 \pm 0.15$ & $+0.11 \pm 0.17$ \\
\hline LeoA & 31326 & 4617 & 1.23 & $-1.74 \pm 0.13$ & $+1.12 \pm 0.44$ & $\ldots$ & $+0.07 \pm 0.39$ & $\ldots$ & $\ldots$ \\
\hline LeoA & 11200 & 4432 & 1.04 & $-1.41 \pm 0.19$ & $\ldots$ & $\ldots$ & $\ldots$ & $\ldots$ & $\ldots$ \\
\hline LeoA & 31582 & 4879 & 1.47 & $-1.62 \pm 0.36$ & $\ldots$ & $\ldots$ & $\ldots$ & $\ldots$ & $\ldots$ \\
\hline LeoA & 31580 & 4814 & 1.36 & $-1.30 \pm 0.15$ & $\ldots$ & $\ldots$ & $\ldots$ & $\ldots$ & $\ldots$ \\
\hline
\end{tabular}

(This table is available in its entirety in machine-readable form.)

of the gas flow during star formation. For example, the narrowness of the distribution can indicate how much the galaxy violates the assumptions of the closed box or leaky box.

Figure 9 shows the metallicity distributions for the three dIrrs. Leo A has the best sampled distribution, followed by SagDIG and then Aquarius. As a result, the quantitative results for Leo A will be the most secure. Within the margins of uncertainty, there is no significant difference between the shapes of the metallicity distributions other than the mean metallicity. The skewness and kurtosis of all three distributions are consistent with each other and with zero. Interestingly, these shapes are more in line with small dSphs, which had a limited star formation lifetime (Kirby et al. 2013). Leo A, Aquarius, and SagDIG all had star formation durations well in excess of several gigayears (Cole et al. 2007, 2014; Weisz et al. 2014).

Deductions concerning chemical evolution from the metallicity distributions can be made more quantitative by using the equations of chemical evolution. Under certain assumptions, the equations predict a stellar metallicity distribution. The assumptions we invoke here are homogeneity (a one-zone model), instantaneous mixing of gas, and instantaneous recycling of stellar ejecta. We invoke the same models considered by Kirby et al. (2011b, 2013). Those models are the leaky box, as discussed above; the pre-enriched model, which is the leaky box that starts with nonzero metallicity; and the accretion model, which allows for gas inflow during star formation.

The leaky box predicts the following metallicity distribution:

$$
\frac{d N}{d[\mathrm{Fe} / \mathrm{H}]} \propto\left(\frac{10^{[\mathrm{Fe} / \mathrm{H}]}}{p_{\mathrm{eff}}}\right) \exp \left(-\frac{10^{[\mathrm{Fe} / \mathrm{H}]}}{p_{\mathrm{eff}}}\right) .
$$

The only free parameter is $p_{\text {eff. }}$. The pre-enriched model (Pagel 1997) predicts a similar metallicity distribution:

$$
\frac{d N}{d[\mathrm{Fe} / \mathrm{H}]} \propto\left(\frac{10^{[\mathrm{Fe} / \mathrm{H}]}-10^{[\mathrm{Fe} / \mathrm{H}]_{0}}}{p_{\mathrm{eff}}}\right) \exp \left(-\frac{10^{[\mathrm{Fe} / \mathrm{H}]}}{p_{\mathrm{eff}}}\right) .
$$

The two free parameters are $p_{\text {eff }}$ and $[\mathrm{Fe} / \mathrm{H}]_{0}$, the initial metallicity of the gas. Lynden-Bell (1975) invented the accretion model (called the best accretion model by LyndenBell and the extra gas model by Kirby et al. 2011b). The infalling gas has zero metallicity, and it falls in at a prescribed rate. The metallicity distribution is described by two transcendental equations that must be solved for the stellar mass fraction, $s$.

$$
\begin{aligned}
{[\mathrm{Fe} / \mathrm{H}](s)=} & \log \left\{p_{\mathrm{eff}}\left(\frac{M}{1+s-\frac{s}{M}}\right)^{2}\right. \\
& \left.\times\left[\ln \frac{1}{1-\frac{s}{M}}-\frac{s}{M}\left(1-\frac{1}{M}\right)\right]\right\} \\
\frac{d N}{d[\mathrm{Fe} / \mathrm{H}]} \propto & \frac{10^{[\mathrm{Fe} / \mathrm{H}]}}{p_{\text {eff }}} \\
& \times \frac{1+s\left(1-\frac{1}{M}\right)}{\left(1-\frac{s}{M}\right)^{-1}-2\left(1-\frac{1}{M}\right) \times 10^{[\mathrm{Fe} / \mathrm{H}] / p_{\text {eff }}} .} .
\end{aligned}
$$

The two free parameters are $p_{\text {eff }}$ and the accretion parameter, $M$, which is the ratio of the final mass to the initial gas mass.

One major assumption of the models is that they assume that the galaxy has completed its star formation. Otherwise, the prediction is only valid for stars below a certain metallicity. For the leaky box, that metallicity is $p_{\text {eff }} \ln \mu^{-1}$. In fact, the model would predict no stars to form above that metallicity, which is the present gas-phase metallicity. About half of the stars in the three dIrrs have metallicities larger than this threshold. Clearly, the dIrrs violate some of the model assumptions. Nonetheless, we fit these models to examine how well their distributions conform to some of the most basic predictions from chemical evolution.

We fit each of these models to each of the observed metallicity distributions. The likelihood that a galaxy's metallicity distribution conforms to a model is

$$
\begin{aligned}
L= & \prod_{i} \int_{-\infty}^{\infty} \frac{d P}{d[\mathrm{Fe} / \mathrm{H}]} \frac{1}{\sqrt{2 \pi} \delta[\mathrm{Fe} / \mathrm{H}]_{i}} \\
& \times \exp \left(-\frac{\left([\mathrm{Fe} / \mathrm{H}]-[\mathrm{Fe} / \mathrm{H}]_{i}\right)^{2}}{2\left(\delta[\mathrm{Fe} / \mathrm{H}]_{i}\right)^{2}}\right) d[\mathrm{Fe} / \mathrm{H}] .
\end{aligned}
$$

In Equation (7), $d P / d[\mathrm{Fe} / \mathrm{H}]$ is the probability distribution of the model, and $[\mathrm{Fe} / \mathrm{H}]_{i}$ and $\delta[\mathrm{Fe} / \mathrm{H}]_{i}$ are the metallicity and error of the $i$ th observed star. We used an MCMC to maximize the likelihood that all of the measured metallicities with errors 
Table 4

Galaxy Properties

\begin{tabular}{|c|c|c|c|c|}
\hline Property & Leo A & Aquarius & SagDIG & Unit \\
\hline \multicolumn{5}{|c|}{ Photometric Properties } \\
\hline Distance & $827 \pm 11$ & $977 \pm 45(2)$ & $1047 \pm 53(3)$ & $\mathrm{kpc}$ \\
\hline$L_{V}$ & $6.6 \pm 1.4(4)$ & $1.7 \pm 0.2(5)$ & $4.6 \pm 1.1(6)$ & $10^{6} L_{\odot}$ \\
\hline$r_{h}$ & $2.15 \pm 0.12$ & $1.10 \pm 0.03(5)$ & $0.91 \pm 0.05(6)$ & $\operatorname{arcmin}$ \\
\hline$r_{h}$ & $517 \pm 29$ & $312 \pm 16(5)$ & $277 \pm 20(6)$ & \\
\hline$M_{*}$ & $3.3 \pm 0.7(7)$ & $1.5 \pm 0.2(7)$ & $1.8 \pm 0.5(7)$ & $10^{6} M_{\odot}$ \\
\hline $\operatorname{SFR}(\mathrm{H} \alpha)$ & $9.3(8)$ & $0(9,10)$ & $8.5(8)$ & $10^{-5} M_{\odot} \mathrm{yr}^{-1}$ \\
\hline SFR(UV) & $6.0(8)$ & $0(11)$ & $7.2(8)$ & $10^{-4} M_{\odot} \mathrm{yr}^{-1}$ \\
\hline \multicolumn{5}{|c|}{ Gas Properties } \\
\hline$M(\mathrm{H} \mathrm{I})$ & $7.4 \pm 0.8(12)$ & $2.2 \pm 0.3(12)$ & $8.3 \pm 1.2(12)$ & $10^{6} M_{\odot}$ \\
\hline$\left\langle v_{\text {helio }}\right\rangle(\mathrm{H} \mathrm{I})$ & $23.7(12)$ & $-140.3(12)$ & $-79.2(12)$ & $\mathrm{km} \mathrm{s}^{-1}$ \\
\hline$\sigma_{v}(\mathrm{H} \mathrm{I})$ & $6.2(12)$ & $6.7(12)$ & $8.2(12)$ & $\mathrm{km} \mathrm{s}^{-1}$ \\
\hline \multicolumn{5}{|c|}{ Stellar Dynamical Properties } \\
\hline$N_{\text {member }}$ & 127 & 25 & 45 & $\ldots$ \\
\hline$\left\langle v_{\text {helio }}\right\rangle$ & $26.2_{-0.9}^{+1.0}$ & $-141.8_{-2.0}^{+1.8}$ & $-78.4 \pm 1.6$ & $\mathrm{~km} \mathrm{~s}^{-1}$ \\
\hline$v_{\mathrm{GSR}}$ & -13.9 & -30.7 & 6.2 & $\mathrm{~km} \mathrm{~s}^{-1}$ \\
\hline$\sigma_{v}$ & $9.0_{-0.6}^{+0.8}$ & $7.8_{-1.1}^{+1.8}$ & $9.4_{-1.1}^{+1.5}$ & $\mathrm{~km} \mathrm{~s}^{-1}$ \\
\hline$M_{1 / 2}^{\mathrm{a}}$ & $3.9 \pm 0.4$ & $1.8_{-0.3}^{+0.4}$ & $2.3_{-0.3}^{+0.1}$ & $10^{7} M_{\odot}$ \\
\hline$\left(M / L_{V}\right)_{1 / 2}{ }^{\mathrm{b}}$ & $12 \pm 3$ & $21_{-4}^{+6}$ & $10 \pm 3$ & $M_{\odot} L_{\odot}^{-1}$ \\
\hline$\left(M_{\mathrm{tot}} / M_{b}\right)_{1 / 2}{ }^{\mathrm{c}}$ & $7.3_{-1.0}^{+1.1}$ & $9.6_{-1.8}^{+2.5}$ & $4.4_{-0.8}^{+1.0}$ & $\cdots$ \\
\hline \multicolumn{5}{|c|}{ Stellar Chemical Properties } \\
\hline$\langle[\mathrm{Fe} / \mathrm{H}]\rangle$ & $-1.67_{-0.08}^{+0.09}$ & $-1.50 \pm 0.06$ & $-1.88_{-0.09}^{+0.13}$ & $\ldots$ \\
\hline$p_{\text {eff }}($ leaky box $)$ & $3.5 \pm 0.4$ & $5.5_{-1.1}^{+1.2}$ & $2.4_{-0.4}^{+0.5}$ & $10^{-2} Z_{\odot}$ \\
\hline$p_{\text {eff }}$ (pre-enriched) & $2.6_{-0.3}^{+0.4}$ & $5.3_{-1.2}^{+1.4}$ & $2.4_{-0.4}^{+0.5}$ & $10^{-2} Z_{\odot}$ \\
\hline$[\mathrm{Fe} / \mathrm{H}]_{0}$ (pre-enriched) & $-2.47_{-0.12}^{+0.10}$ & $<-2.28^{\mathrm{d}}$ & $<-3.99^{\mathrm{d}}$ & $\ldots$ \\
\hline$\Delta(\ln P)(\text { pre-enriched })^{\mathrm{e}}$ & 12.13 & -1.68 & -1.72 & $\ldots$ \\
\hline$p_{\text {eff }}$ (accretion) & $3.2 \pm 0.3$ & $4.4_{-0.7}^{+0.8}$ & $2.1_{-0.3}^{+0.4}$ & $10^{-2} Z_{\odot}$ \\
\hline$M$ (accretion) & $6.0_{-2.0}^{+3.2}$ & $7.1_{-3.8}^{+6.2}$ & $2.3_{-0.9}^{+1.9}$ & $\ldots$ \\
\hline$\Delta(\ln P)(\text { accretion })^{\mathrm{e}}$ & 11.03 & 0.37 & -1.19 & $\ldots$ \\
\hline
\end{tabular}

Notes.

${ }^{a}$ Mass within the half-light radius, calculated as $M_{1 / 2}=4 G^{-1} \sigma_{v}^{2} r_{h}$ (Wolf et al. 2010).

${ }^{\mathrm{b}}$ Mass-to-light ratio within the half-light radius.

${ }^{\mathrm{c}}$ Total (dynamical) mass divided by baryonic mass $\left(M_{b}=M_{*}+M(\mathrm{H} \mathrm{I})\right)$ within the half-light radius. The calculation assumes that half of the stellar mass and half of the gas mass is located within the half-light radius.

d Upper limit with $95 \%$ confidence.

${ }^{\mathrm{e}}$ Logarithm of the strength with which the pre-enriched or accretion model is favored over the leaky box model. Negative values of $\Delta(\ln P$ ) indicate that the model is disfavored.

References. (1) Tammann et al. (2011). (2) Cole et al. (2014). (3) Momany et al. (2005). $L_{V}$ and $r_{h}$ based on surface brightness profiles from (4) de Vaucouleurs et al. (1991), (5) McConnachie et al. (2006), and (6) Lee \& Kim (2000). Both values are updated for the distances adopted here. $L_{V}$ is corrected for extinction based on Schlafly \& Finkbeiner (2011). (7) Based on stellar mass-to-light ratios from Woo et al. (2008). (8) Karachentsev \& Kaisina (2013). (9) van Zee (2000). (10) Hunter \& Elmegreen (2004). (11) Hunter et al. (2010). (12) Measured from the H I maps of Hunter et al. (2012). H I masses updated for the distances adopted here. The uncertainty in $M(\mathrm{H} \mathrm{I})$ incorporates error on the $21 \mathrm{~cm}$ flux-assumed to be $11 \%$-and uncertainty in distance.

less than 0.5 dex conform to the model. The lengths of the MCMC chains were $10^{3}$ for the leaky box and $10^{5}$ for the other two models. Table 4 gives the best-fitting values and their $68 \%$ confidence intervals.

We also computed the corrected Akaike information criterion (AICc; Akaike 1974; Sugiura 1978, p. 13), which quantifies how well one model fits over another. It penalizes models with more free parameters. This is especially important for the pre-enriched and accretion models because they are both generalizations of the leaky box model. Therefore, it is not possible for the leaky box model, which has only one free parameter, to fit better than either of the other two models. The
AICc allows for the leaky box model to be favored even if its formal likelihood is lower. The probability that one model fits better than another is $\Delta(\ln P)=\Delta \mathrm{AICc} / 2$. Table 4 lists the values of $\Delta(\ln P)$ for the pre-enriched and accretion models as compared to the leaky box model. Negative values mean that the leaky box model is preferred over the alternative model.

Formally, each galaxy prefers a different model. Leo A favors the pre-enriched model. Figure 9 shows that the preenriched model does the best job at fitting the metal-poor end of the distribution. However, the accretion model fits the peak better. This ambivalence is reflected in the small difference between AICc for the two models. The accretion model has a 


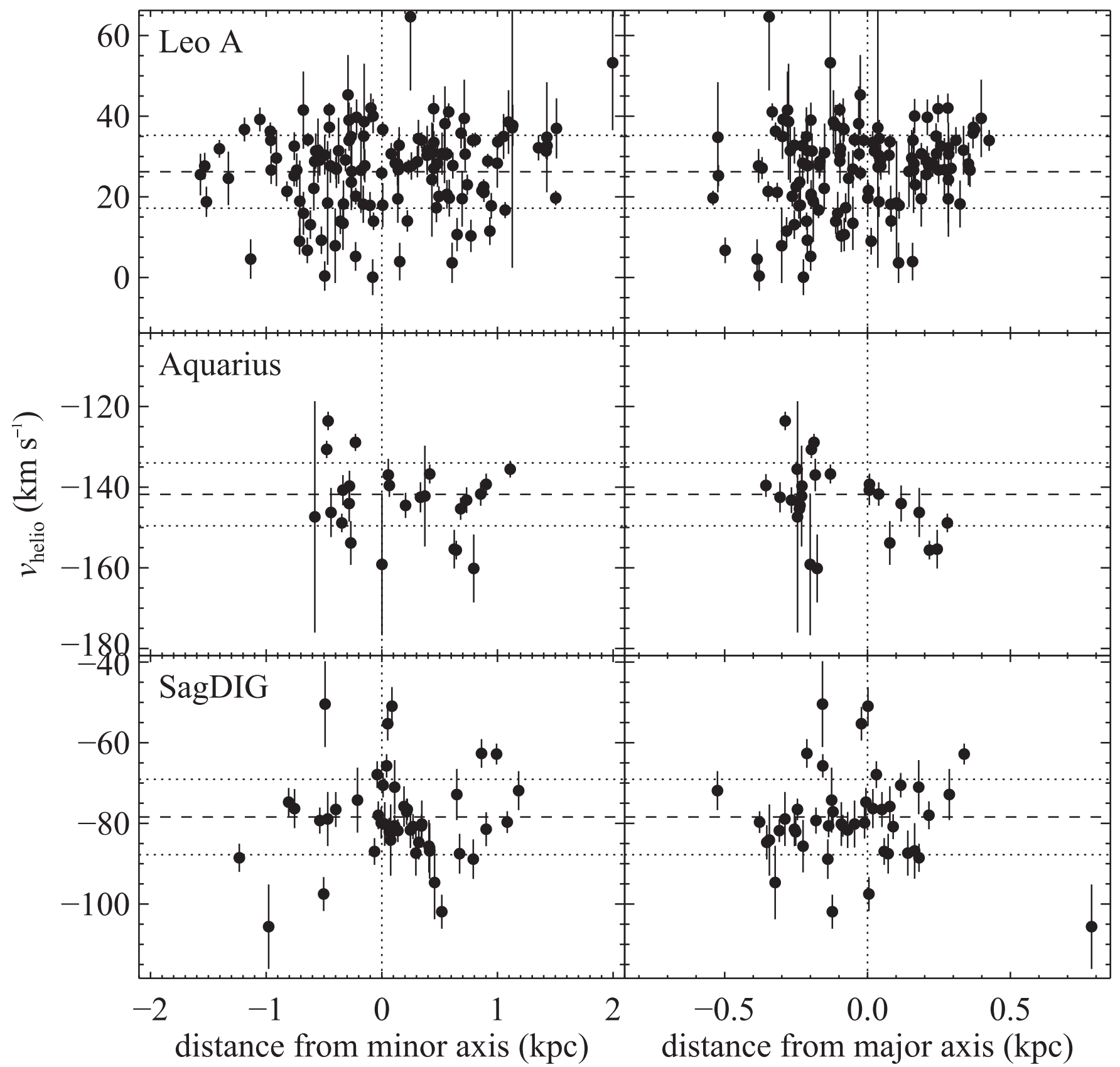

Figure 6. Heliocentric radial velocities of member stars as a function of distance from the minor axis (left) and major axis (right) of their respective galaxy. Rotation about the minor axis is more common. However, stellar rotation is not apparent in either projection. The horizontal dashed lines show the mean velocities, and the horizontal dotted lines show the $1 \sigma$ velocity dispersions.

probability of 0.33 of fitting the metallicity distribution of Leo A relative to the pre-enriched model. Thus, neither model is strongly preferred, but they are both strongly preferred relative to the leaky box model.

Aquarius and SagDIG show no evidence for requiring any additional complication beyond the leaky box. Formally, Aquarius prefers the accretion model. However, the difference of AICc between the accretion and leaky box models is negligible. For both models, the best-fit value of $[\mathrm{Fe} / \mathrm{H}]_{0}$ for the pre-enriched model was extremely low. As a result, the preenriched model is nearly indistinguishable from the leaky box model. Although the data do not justify an additional free parameter beyond the leaky box, the metallicity distributions are not as well sampled as Leo A. Our result might be different with more measurements.

Again, the dIrrs violate some of the assumptions of these chemical evolution models. In particular, they have not run out of gas, so we should not be fitting the distributions of the more metal-rich stars. Furthermore, all three dIrrs are late-forming. They must have acquired gas or somehow made their gas available for star formation long after their dark matter halos collapsed. As a result, any gas accretion that might have powered star formation almost certainly does not follow the functional form described by Equation (6). 


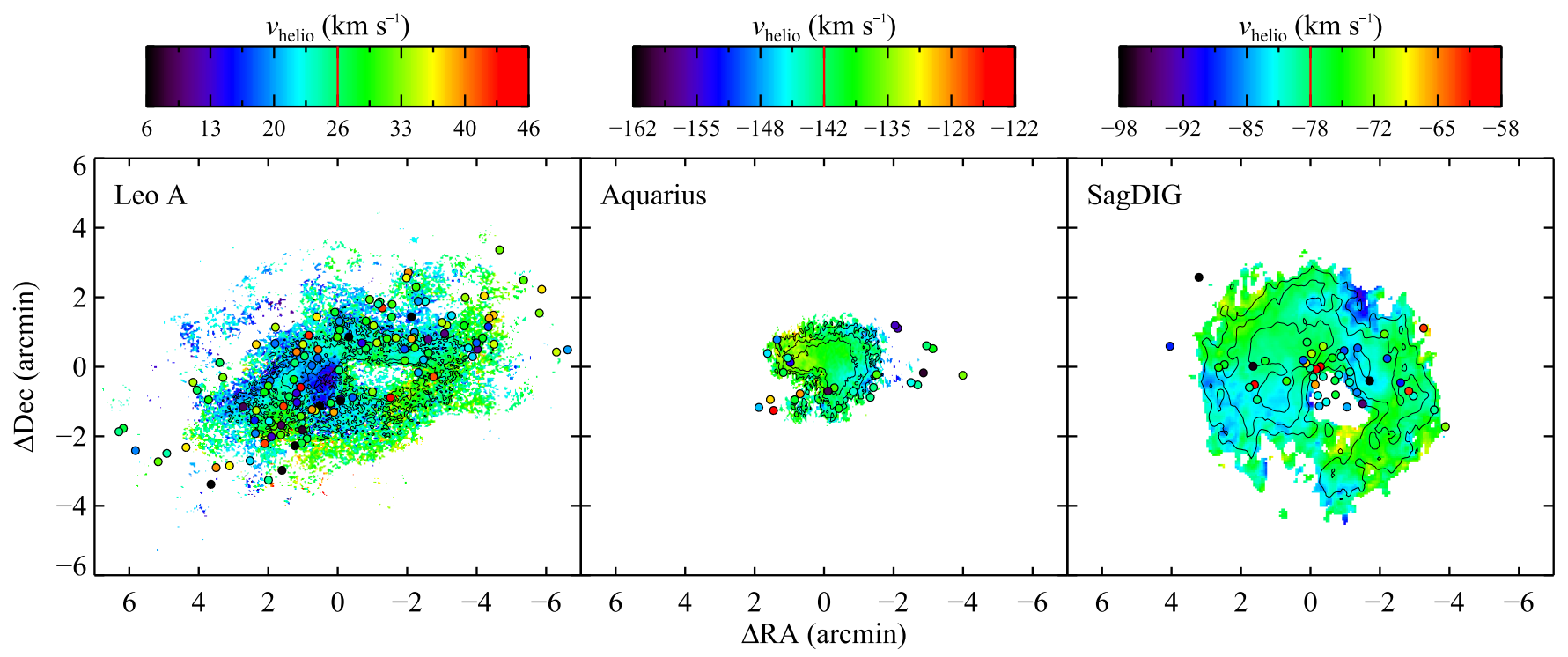

Figure 7. Kinematics of H I gas in Leo A, Aquarius, and SagDIG, as observed by LITTLE THINGS (Hunter et al. 2012). The gas distribution is color-coded according to the velocity scale shown at the top of each plot. A red line in the velocity scale indicates the average velocity, $\left\langle v_{\text {helio }}\right\rangle$, of the stars. The black contours indicate the flux of the $21 \mathrm{~cm}$ measurements. The contour levels are 4,8 , and $16 M_{\odot} \mathrm{pc}^{-2}$ for Leo A and 1.25, 2.5, and $5 M_{\odot} \mathrm{pc}^{-2}$ for Aquarius and SagDIG. The stars are shown as black-outlined circles. The color of the circle shows the star's velocity on the same color scale as the gas.

\subsubsection{Correction for Selection Bias}

Red giant lifetimes are much less than a Hubble time, and they vary as a function of stellar metallicity and mass. As a result, the observed distribution of red giant metallicities is not guaranteed to be an exact representation of the actual mass fraction of heavy elements in a stellar population that spans a wide range of ages. Even in the absence of any color selection, the varying red giant lifetimes bias the observed metallicity distributions. E. M. Manning \& A. A. Cole (2016, in preparation) are investigating the relationship between the observational sample selection of red giant stars and the actual underlying distribution of stellar metallicities. They use synthetic RGBs based on PARSEC isochrones (Bressan et al. 2012) to estimate the expected number distribution of red giants, representing various populations in the spectroscopic sample as a function of both age and metallicity, allowing for scaling dictated by the SFH. The net result is a variation on the order of $20 \%$ between the oldest, most metalpoor stars and the youngest, most metal-rich stars. Manning \& Cole will present stellar number distributions scaled by the approximate over- or underrepresentation of giants as a function of metallicity for predominantly intermediate-age populations, like those in Leo A and Aquarius.

We calculated scaling factors for each bin in the metallicity histograms (Figure 9) based on the HST-derived SFHs and age-metallicity relations of both Leo A and Aquarius (Cole et al. 2014). The shaded histograms in Figure 10 show the expected number of stars for each metallicity bin of the entire stellar population, whereas the unshaded histograms show the observed number of red giants in each metallicity bin. The SFHs were used to estimate the mean population age, but the correction factors do not depend strongly on the detailed variation of SFR with time. We did not compute corrections for SagDIG because the available photometry is not as deep as for Leo A or Aquarius.

With the corrections from E. M. Manning \& A. A. Cole (2016, in preparation), we found that the corrected mean metallicity of Leo A is lower than the observed distribution by
0.07 dex, whereas the mean metallicity of Aquarius is unchanged. The corrected histograms for both dIrrs are slightly less concentrated toward the peak than the observed histograms.

The changes in the mean and shape of the metallicity distribution will affect the parameters derived for the chemical evolution models. They could also affect which model is preferred. In the case of Leo A, the effective yields would be lower by an amount comparable to the shift in mean metallicity. The slightly less peaked distribution also would cause the pre-enriched model to have an even higher likelihood relative to the accretion model. However, the corrections still do not allow for the leaky box model without pre-enrichment. The corrections in Aquarius are not severe enough to significantly affect the parameters or likelihoods of the chemical evolution models.

Most dIrrs have not yet been subjected to scrutiny of the red giant selection effect. In order to compare galaxies on level footing, we restrict our analysis to the uncorrected metallicity distributions. None of the figures and tables except Figure 10 reflect these corrections.

\subsection{Metallicity Gradients}

The metallicity of stars in a galaxy often decreases with distance from the center of the galaxy. A variety of scenarios could give rise to a radially decreasing metallicity gradient. For instance, low-metallicity gas that falls onto the galaxy would likely have some angular momentum. That gas would end up in the outer regions of the galaxy, diluting the metallicity of any gas that happened to be forming stars. Alternatively, if the galaxy loses gas, it will lose it most readily from the outer parts of the galaxy, where the gravitational potential is the weakest. As a result, later star formation is likely to come from gas surviving at the center of the galaxy.

Figure 11 shows the radial metallicity gradients in the three dIrrs. All of the gradients are negative, in line with the preceding arguments and consistent with most other dwarf galaxies. However, the gradients are shallow. The slopes in 


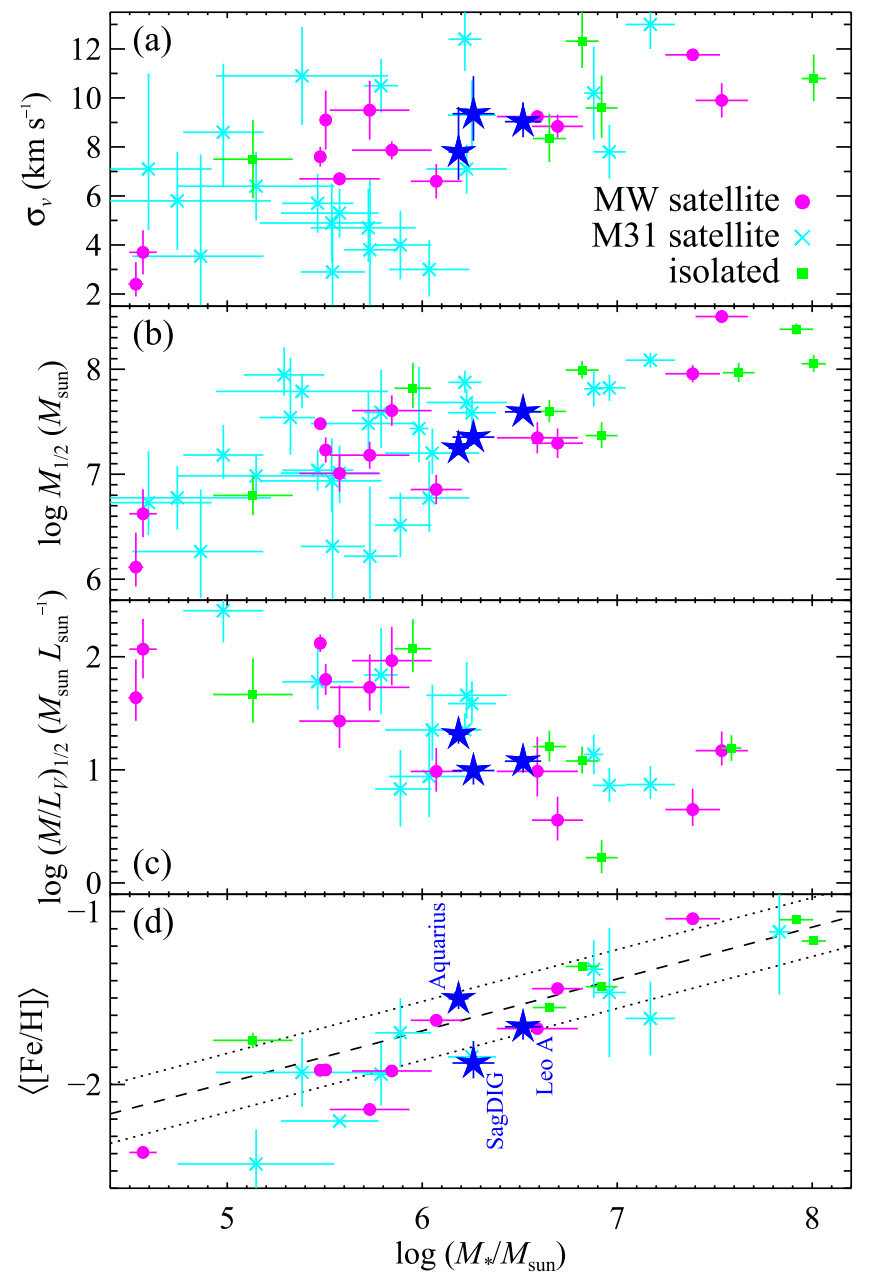

Figure 8. Trend with stellar mass of (a) velocity dispersion, (b) mass within the half-light radius, (c) mass-to-light ratio within the half-light radius, and (d) average metallicity. MW satellite galaxies are shown as magenta circles, and isolated galaxies in and around the Local Group are shown as green squares. The dashed line in panel (d) shows the mass-metallicity relation from Kirby et al. (2013). The dotted lines show the 0.17 dex scatter about the relation. The three isolated galaxies presented in this paper are shown as blue five-pointed stars. For other isolated galaxies, the dynamical quantities are taken from Simon \& Geha (2007, Leo T), Fraternali et al. (2009, Tucana), and Kirby et al. (2014, others). For the MW satellites, the dynamical quantities are taken from Simon \& Geha (2007), Mateo et al. (2008), Adén et al. (2009), Koch et al. (2009), Walker et al. (2007, 2009a, 2009b, 2009c), Koposov et al. (2011), and Frinchaboy et al. (2012). The dynamical quantities for the M31 satellites are from Tollerud et al. (2012, 2013) and Collins et al. (2013). All metallicities are from Kirby et al. (2013).

Leo A and Aquarius are only marginally distinct from zero, and the slope in SagDIG is effectively zero.

A rigorous analysis of the metallicity gradient should sample the metallicities of stars out to many half-light or core radii, ideally out to the tidal radius. Our survey samples much of the extent of Aquarius and most of SagDIG. However, Leo A contains quite a few stars beyond the extent of our spectroscopic sample. Therefore, our results are strictly applicable only to the range of stars we have observed. Some galaxies, especially dSphs, can have changes in the slopes of their metallicity gradients that would not be detected in stellar samples with limited angular extent (e.g., Battaglia et al. 2011).

Leaman et al. (2013) found that the radial metallicity gradients in dIrrs are usually shallower than dSphs. Indeed, Kirby et al. (2011b) found rather steep gradients in some MW
dSphs, though some of their spectroscopic samples were limited to about 10 arcmin from the center of the dSph. Leaman et al. further found that the gradients were the least steep in galaxies with more rotational support. For example, the three highest-luminosity dSph satellites of M31 (NGC 147, NGC 185, and NGC 205; see Kormendy \& Bender 2012) have metallicity gradients that monotonically steepen as their rotational support $\left(v_{\text {rot }} / \sigma_{v}\right)$ decreases (Geha et al. 2006, 2010; Koleva et al. 2009). As Leaman et al. (2013) pointed out, rotation induces an angular momentum barrier that prevents gas and consequently star formation from concentrating in the center of the galaxy. As a result, chemical evolution and the increase of metallicity occur at similar rates at all radii (see Schroyen et al. 2011 for galaxy simulations that support this hypothesis). Alternatively, radial migration could flatten any preexisting metallicity gradient.

We did not detect rotational support in the stellar populations of Leo A, Aquarius, or SagDIG (Section 4.2). However, our observations only place limits on the amount of rotation. They do not conclusively rule out rotation. This is especially true for SagDIG, which shows a rather circular distribution of $\mathrm{H}$ I gas, suggesting that we could be viewing the galaxy face-on. Thus, our observations neither support Leaman et al.'s finding that metallicity gradients become shallow with increasing $v_{\text {rot }} / \sigma_{v}$ nor provide evidence against it. Larger samples could help tighten the constraints on both $v_{\text {rot }} / \sigma_{v}$ and the metallicity gradients.

\subsection{The Age-Metallicity Relation}

The broadband colors of red giants depend on their ages and metallicities. Therefore, we can couple the photometry of the stars with our spectroscopic measurements of their metallicities. We used Yonsei-Yale isochrones (Demarque et al. 2004) for this purpose. First, we isolated the two sets of isochrones that bordered a given metallicity. We interpolated each isochrone in metallicity space to create a set of isochrones at the exact metallicity of each star. Then, we isolated the two isochrones that bordered the $I_{0}$ magnitude and $(V-I)_{0}$ color of the star in question. We linearly interpolated between the two isochrones to estimate the age of that star. For stars where we measured $[\alpha / \mathrm{Fe}] \geqslant+0.3$, we used the $[\alpha / \mathrm{Fe}]=+0.3$ isochrones. For stars where we measured $[\alpha / \mathrm{Fe}] \leqslant 0.0$, we used the $[\alpha / \mathrm{Fe}]=0.0$ isochrones. For other stars, we used linear interpolations of the two isochrone sets.

When we measured metallicities from the DEIMOS spectra, we used photometry to fix the surface gravity and provide a best guess at $T_{\text {eff }}$. In this process, we used Yonsei-Yale isochrones with an assumed age of 14 Gyr. Thus, it might seem circular to derive ages from these metallicities. However, the photometric temperature is used only as a first guess. The spectrum determines the final temperature. Furthermore, the color-temperature relations are not particularly sensitive to age. For example, consider a star with $M_{V}=-2.3$ and $V-I=1.2$. The photometric temperature for this star would be $4400 \mathrm{~K}$ at 2 Gyr and $4477 \mathrm{~K}$ at $14 \mathrm{Gyr}$. Even if we adopted strictly photometric temperatures in the metallicity determinations, the difference in $[\mathrm{Fe} / \mathrm{H}]$ between those two temperatures would be less than 0.1 dex (e.g., Kirby et al. 2010). In contrast, the metallicities at those ages would be $[\mathrm{Fe} / \mathrm{H}]=-1.29$ and -1.91 , respectively. Hence, the spectroscopic metallicity does have the power to determine ages, even if the spectroscopic metallicities were based partly on photometric temperatures. 

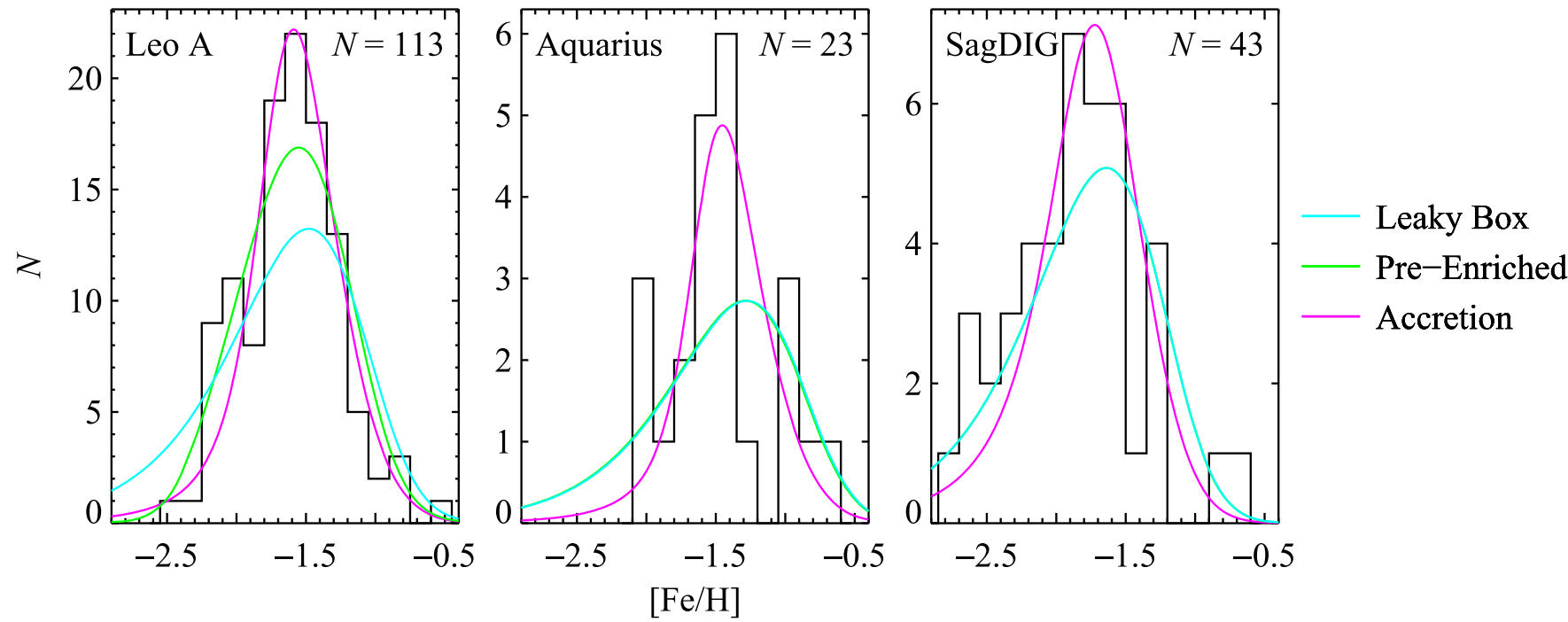

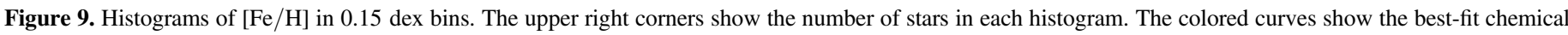
evolution models.

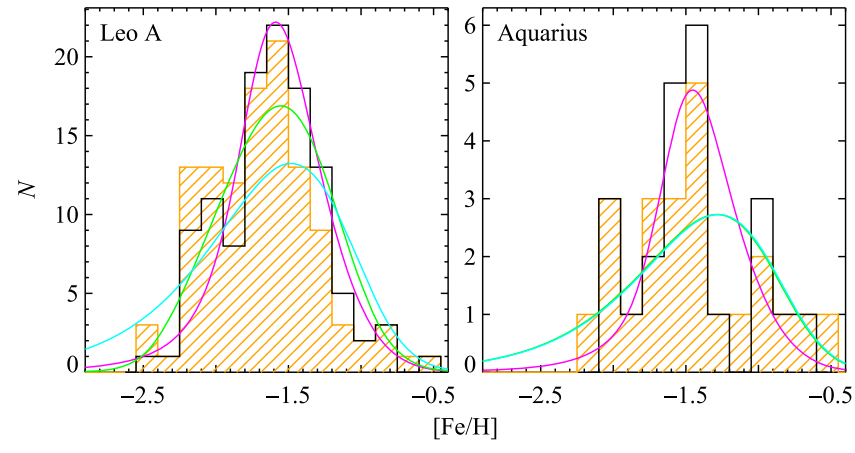

Figure 10. Metallicity distributions for Leo A and Aquarius. The unshaded black histograms are observed (same as Figure 9). The shaded orange histograms are corrected for RGB selection bias, as described in Section 5.1.1. The curves show the same chemical evolution models as Figure 9.

To estimate errors, we resampled the star's color, magnitude, and metallicity. For example, in one realization, we perturbed the magnitude by $R \delta I_{0}$, where $R$ is a random number drawn from a Gaussian distribution with unit width and $\delta I_{0}$ is the uncertainty on the magnitude. The metallicity and color were perturbed in a similar fashion, and we recomputed the age for this combination of values. We repeated this process $10^{3}$ times. We took the uncertainty on the age to be the standard deviation of all of the trials.

Figure 12 shows metallicities as a function of the resulting ages. Measurements with uncertainties larger than $4 \mathrm{Gyr}$ are excluded. Some ages are younger than zero or older than the age of the universe. These measurements reflect measurement uncertainty or imperfect isochrone models. Even so, the metallicity mostly increases with time in all cases, as expected for most galaxies.

Cole et al. (2007) derived a very shallow age-metallicity relation for Leo A. They found that a model with $[\mathrm{Fe} / \mathrm{H}]=$ -1.4 , constant with time, fit the CMD well. We also found a shallow age-metallicity relation, but it turns up within the last 5 Gyr.

Cole et al. (2014) also measured the photometric agemetallicity relation for Aquarius. They found a positive slope up to $6 \mathrm{Gyr}$ ago, followed by virtually no metallicity evolution thereafter. In contrast, we found a steep age-metallicity

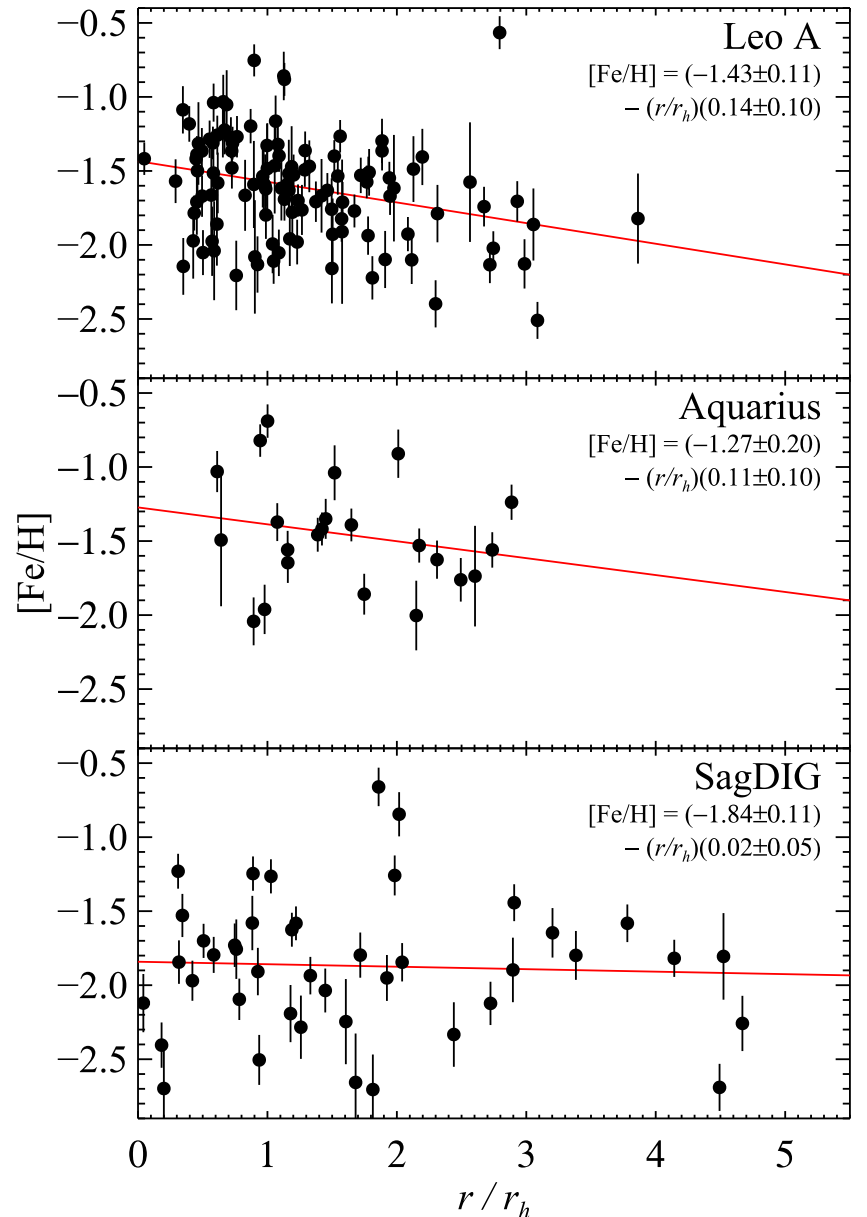

Figure 11. Relation between metallicity and radial distance from the center of the galaxy in units of the half-light radius. The red lines are least-squares linear regressions whose parameters are shown in the upper right corner of each panel.

relation. However, the oldest age we measured is between 5 and 6 Gyr. We attribute the difference in results to two factors. First, we used ground-based photometry, whereas Cole et al. used HST photometry, which is more accurate. Second, 


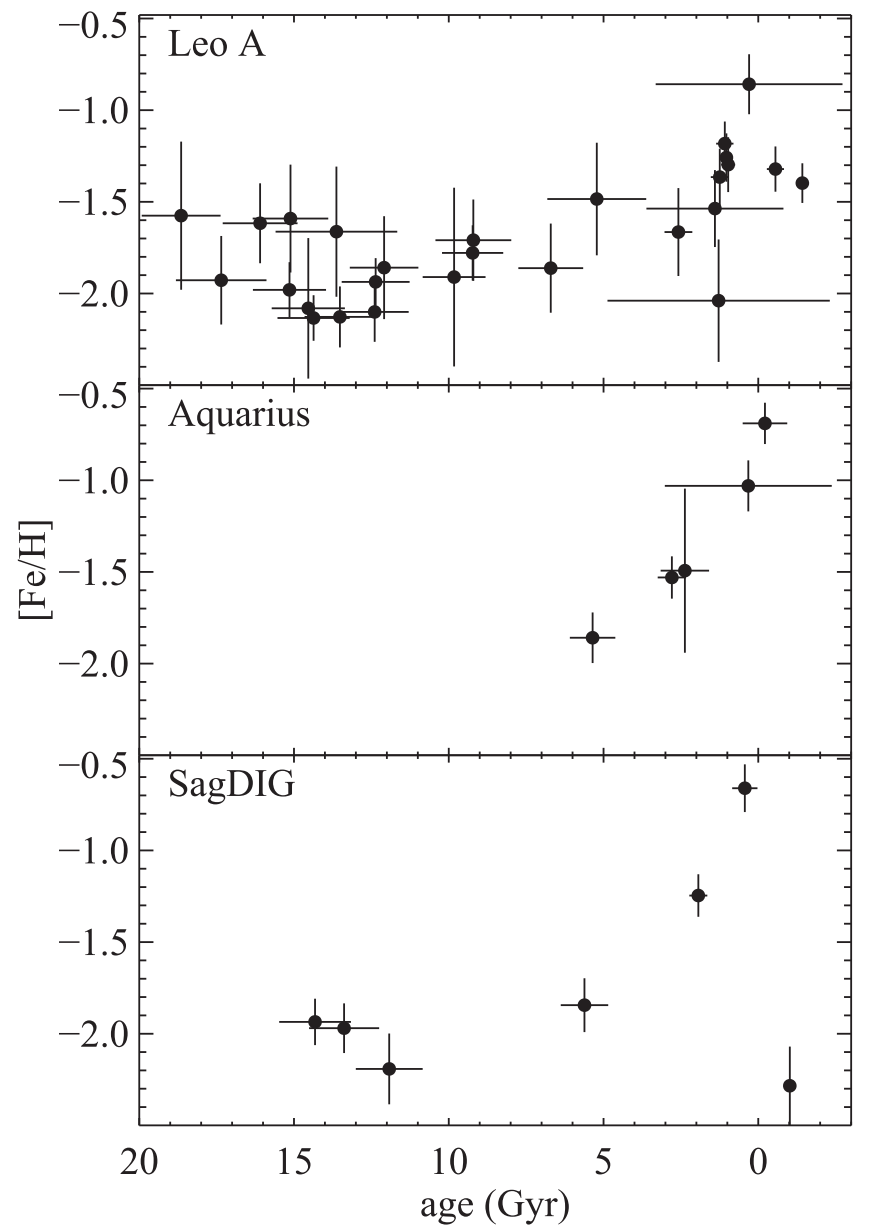

Figure 12. Relation between metallicity and age for individual stars.

Cole et al. fit entire stellar populations, whereas we fit individual stars. Third, we have access to the spectroscopic metallicities. In principle, this information should allow a fairly precise measurement of age, but in practice, random errors in the photometry and systematic errors in the isochrones limit the precision of the measurement.

\section{4. $\alpha$ Elements}

Chemical abundance ratios provide a method of estimating SFHs complementary to CMD fitting. The trend of the $[\alpha / \mathrm{Fe}]$ ratio with $[\mathrm{Fe} / \mathrm{H}]$ is sensitive to the changing ratio of Type II to Type Ia supernovae with time. A numerical chemical evolution model - as opposed to the analytic models in Section 5.1-can predict the trend of $[\alpha / \mathrm{Fe}]$ versus $[\mathrm{Fe} / \mathrm{H}]$ for an assumed $\mathrm{SFH}$ (e.g., Matteucci \& Greggio 1986; Kirby et al. 2011a).

Figure 13 shows the trend of $[\alpha / \mathrm{Fe}]$ with $[\mathrm{Fe} / \mathrm{H}]$ for the three dIrrs. The MW satellite Sculptor, a dSph, is shown for comparison. The scarcity of points and the measurement uncertainties limit our ability to draw strong conclusions about the chemical evolution of the dIrrs. For example, fitting chemical evolution models in the style of Kirby et al. (2011b) would not be informative. Regardless, it is worth pointing out that this is by far the largest sample of $[\alpha / \mathrm{Fe}]$ measurements in dIrrs. ${ }^{9}$

\footnotetext{
9 However, it is not the largest sample outside of the MW system. See Vargas et al. $(2014 \mathrm{a}, 2014 \mathrm{~b})$ for $[\alpha / \mathrm{Fe}]$ measurements in M31 and its satellites.
}

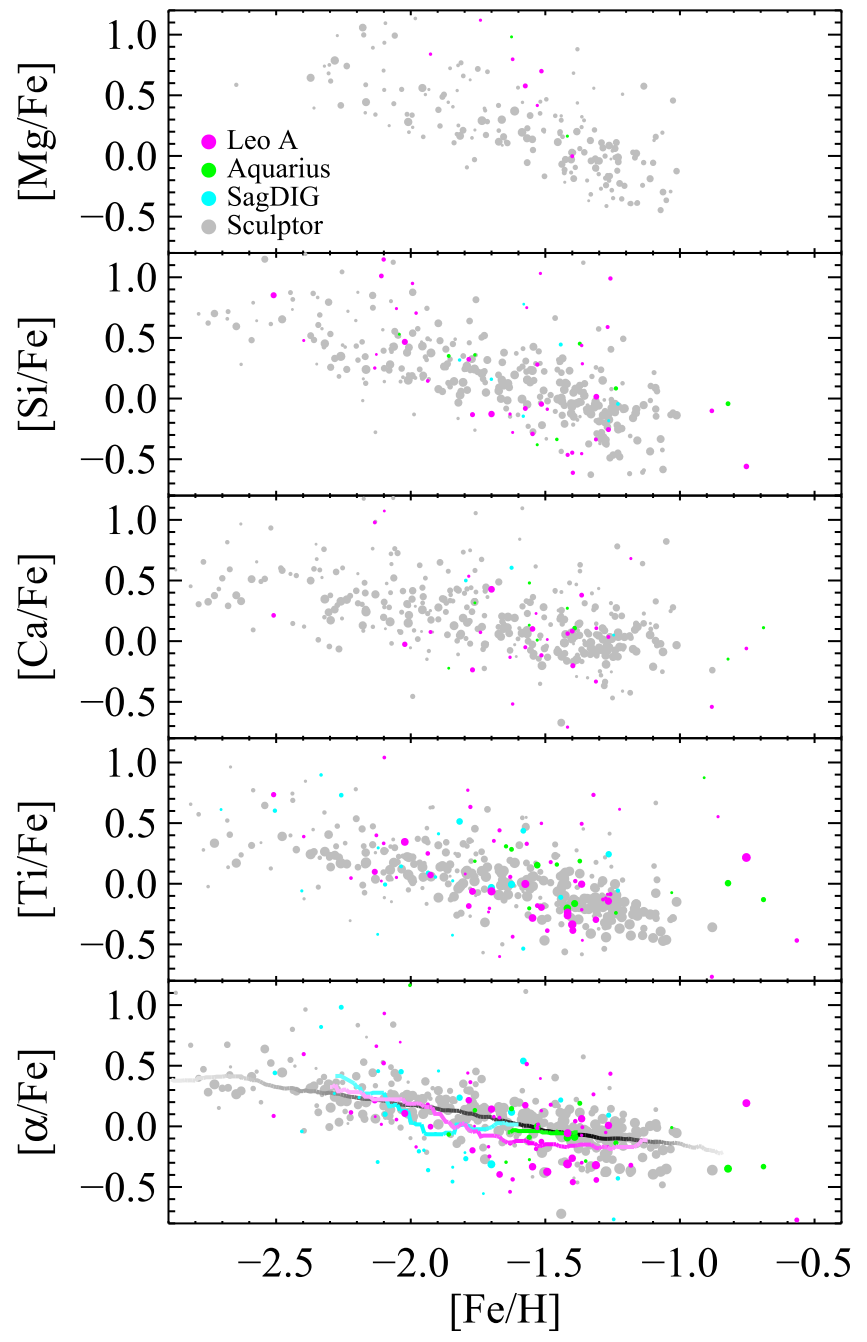

Figure 13. Trend of abundance ratios with metallicity. The three dIrrs are shown in color. For comparison, the Sculptor dSph is shown in gray (data from Kirby et al. 2009, 2010). Circle size is inversely proportional to measurement uncertainty, where the largest circles have uncertainties of about 0.1 dex in each dimension, and the smallest circles (barely visible) have uncertainties of about $0.5 \mathrm{dex}$. The bottom panel shows the average $[\alpha / \mathrm{Fe}]$ ratio, including moving averages in the window of $\Delta[\mathrm{Fe} / \mathrm{H}]=0.5$. The weight of the lines representing the moving averages reflects the number of stars contributing to the average at each $[\mathrm{Fe} / \mathrm{H}]$.

However, the similarity of the dIrrs to Sculptor is interesting, especially in the bottom panel, which shows the average of the $\alpha$ elements. For the most part, the dIrr measurements fall within the envelope defined by Sculptor. Some of the highly uncertain measurements scatter upward to $[\alpha / \mathrm{Fe}]>+0.5$. These measurements are simply uncertain, and they probably do not necessarily reflect the presence of extremely $\alpha$-enhanced stars.

However, some of the more secure measurements in the dIrrs lie on the low side or even entirely below the Sculptor distribution. The moving averages for the dIrrs-especially Leo A-lie slightly below that of Sculptor at $[\mathrm{Fe} / \mathrm{H}]>-1.9$. The underabundance of $\alpha$ elements is most easily interpreted as an SFH that is more extended than in Sculptor. The longer time allows more Type Ia supernovae to explode, lowering the $[\alpha / \mathrm{Fe}]$ ratio. Reassuringly, the photometrically measured SFHs for the dIrrs are significantly more extended than Sculptor. Whereas star formation persisted for 8 Gyr or more in the dIrrs, Sculptor formed no stars after 10 Gyr ago (Revaz et al. 2009; Weisz et al. 2014). 


\section{SUMMARY AND DISCUSSION}

We have presented a comprehensive spectroscopic analysis of the stellar populations in the three dIrrs Leo A, Aquarius, and SagDIG. These galaxies share several properties. First, they have similar stellar masses, spanning a range of (1.5-3.3) $\times 10^{6} M_{\odot}$. Second, they are isolated. The dwarf galaxies nearest to them are hundreds of kiloparsecs away, and the nearest large galaxies (the MW, M31, and M33) are at least $600 \mathrm{kpc}$ away. Second, they formed the majority of their stars well into the lifetime of the universe. Deep HST CMDs show that Leo A and Aquarius formed 80\%-90\% of their stars more recently than 6 and $8 \mathrm{Gyr}$ ago, respectively (Cole et al. 2007, 2014). Although SagDIG does not yet have HST imaging that reaches the MSTO, the CMD of the more luminous stars indicates a similar SFH but with a possibly larger fraction of ancient stars (Weisz et al. 2014).

Their similarities make it even more interesting to study their subtle differences. For example, dwarf galaxies obey a very tight relation between stellar mass and stellar metallicity (Kirby et al. 2011b, 2013). Both gas-rich dIrrs and gas-poor dSphs all fall on the same relation, with no measurable difference between them. The three dIrrs we have studied fall within 1.6 standard deviations of the relation. However, the two most gasrich galaxies, Leo A and SagDIG, fall below the line, whereas Aquarius lies above it. This result is consistent with the expectations of simple models of chemical evolution. As galaxies evolve, they become metal-rich and gas-poor. Hence, gas-rich galaxies have not yet become metal-rich.

The dynamical properties of the three dIrrs are in line with other dwarf galaxies. Their velocity dispersions, masses, and mass-to-light ratios follow the same trend as other Local Group galaxies. Table 4 shows these properties. One quantity of particular interest is the ratio of total mass to the baryonic mass, including stars and $\mathrm{H}$ I gas. Aquarius has the highest ratio. It is easy to imagine that Aquarius appears similar to future versions of Leo A and SagDIG. If the latter two galaxies continue to turn gas into stars, their gas masses and SFRs will decrease. Their dark-to-baryonic mass ratios will increase along with their gas-phase and average stellar metallicities. The final states of Leo A and SagDIG will contain more stellar mass than Aquarius because they have more gas available for star formation. Other than that, they seem to be less evolved-but slightly more massive - versions of Aquarius.

The observed RGB stars do not show any sign of rotation in any of the three dIrrs. As Wheeler et al. (2015) concluded, dIrrs do not seem to transition to dSphs by eliminating well-ordered rotation. Instead, the lowest-mass dwarf galaxies form dynamically hot, supported by dispersion rather than rotation. It is notable that the $\mathrm{H}$ I gas in Aquarius does rotate. In fact, the structure and motions of gas in all of the galaxies have little relation to the old stellar populations. Whereas the gas is clumpy and exhibits small-scale velocity structure, the stellar distribution is smooth. The only similarities between the gas and the old stars are the average velocities and the velocity dispersions. The similarity of velocity dispersions supports the conclusion that the gas is reacting to the galaxies' gravitational potentials rather than hydrodynamics.

The gold standard of measuring SFHs is space-based photometry deep enough to reach the old MSTO. Nonetheless, we have employed complementary techniques to evaluate the ages of stars. First, we fit the ages of stars to model isochrones based on their spectroscopic metallicities. Although the details of the age-metallicity relation do not match the photometrically derived SFHs, we found a monotonic slope in the relation such that younger stars are more metal-rich. Second, we qualitatively described the SFH as compared to the Sculptor dSph, a satellite of the MW, by comparing the $[\alpha / \mathrm{Fe}]$ distributions. On average, the dIrr stars have lower $[\alpha / \mathrm{Fe}]$ at a given $[\mathrm{Fe} / \mathrm{H}]$ than Sculptor. This distinction indicates more of an influence of Type Ia supernovae in the dIrrs than in Sculptor. This result is in agreement with the photometric SFHs because longer star formation durations allow for more Type Ia supernovae.

It is also worthwhile to compare these dIrrs with dIrrs of different masses. Two dIrrs with detailed studies are WLM (Leaman et al. 2012, 2013) and NGC 6822 (Swan et al. 2016), which have stellar masses of $4.5 \times 10^{7} M_{\odot}$ and $1.7 \times 10^{8} M_{\odot}$ (Woo et al. 2008), both considerably more massive than the dIrrs studied in our work. Leaman et al. (2012) presented a rich dynamical data set for WLM. They clearly detected stellar rotation, even though $v_{\text {rot }} / \sigma_{v} \sim 1 .^{10}$ The gas has a similar rotation curve but a velocity dispersion smaller by a factor of 6 . Therefore, WLM seems to have experienced dynamical evolution markedly different from the three dIrrs in our sample. Leaman et al. suggested that giant molecular clouds inflated the velocity dispersion of the stars over time, and they found that the metallicity distribution of WLM, like Leo A, does not conform to a leaky box. Swan et al.'s (2016) spectroscopic study of NGC 6822 tells a similar story. Over time, the stars became dynamically heated, such that the younger stars have lower velocity dispersions than the older stars. Similar to Aquarius, the gas in NGC 6822 rotates, whereas the stars show only scant evidence for rotation (also see Kirby et al. 2014). Like WLM, the younger, more metalrich stars can be found closer to the center.

Our study, as well as other studies of Local Group dIrrs, paints a picture of galaxies tenuously forming stars. Although Leo A, Aquarius, and SagDIG first started forming stars near the beginning of the universe, they managed to form very few stars for billions of years. They experienced low, simmering SFRs since they resumed star formation. Small events could disrupt the gas distribution of the entire galaxy. At any given time, the gas appears in disordered clumps, giving rise to star formation sporadic in both time and position.

Despite teetering at the threshold of the ability to form stars, the galaxies managed to evolve chemically for many gigayears. Their increase in metallicity was slow $\left(0.05-0.30 \mathrm{dex} \mathrm{Gyr}^{-1}\right)$, but they managed to maintain that pace for up to $8 \mathrm{Gyr}$. Furthermore, they follow clear dynamical and chemical trends with stellar mass. The consistent outcomes of these galaxies despite such a tenuous existence suggest that they are perhaps not so fragile after all.

We thank Alan McConnachie, Nobuo Arimoto, and Mike Irwin for kindly sharing their photometry catalog for Aquarius. We also thank Yazan Momany and his collaborators for sharing their photometry catalog of SagDIG. Deidre Hunter provided kind assistance with the LITTLE THINGS $21 \mathrm{~cm}$ images. We acknowledge support from the National Science Foundation through grant 1614081.

\section{Facility: Keck:II (DEIMOS).}

\footnotetext{
${ }^{10}$ Wheeler et al. (2015) found a lower value for $v_{\text {rot }} / \sigma_{v}$, but they also found that WLM exhibited clear stellar rotation. Furthermore, Leaman et al. (2012) corrected for asymmetric drift, which led to a larger value of $v_{\text {rot }}$.
} 


\section{REFERENCES}

Adén, D., Wilkinson, M. I., Read, J. I., et al. 2009, ApJL, 706, L150 Akaike, H. 1974, ITAC, 19, 716

Allsopp, N. J. 1978, MNRAS, 184, 397

Anders, E., \& Grevesse, N. 1989, GeCoA, 53, 197

Battaglia, G., Tolstoy, E., Helmi, A., et al. 2011, MNRAS, 411, 1013 Beccari, G., Bellazzini, M., Fraternali, F., et al. 2014, A\&A, 570, A78 Berg, D. A., Skillman, E. D., Marble, A. R., et al. 2012, ApJ, 754, 98 Bernard, E. J., Gallart, C., Monelli, M., et al. 2008, ApJL, 678, L21 Bernard, E. J., Monelli, M., Gallart, C., et al. 2009, ApJ, 699, 1742 Bressan, A., Marigo, P., Girardi, L., et al. 2012, MNRAS, 427, 127 Brown, W. R., Geller, M. J., Kenyon, S. J., \& Kurtz, M. J. 2007, ApJ, 666, 231 Cohen, J. G., Persson, S. E., \& Frogel, J. A. 1978, ApJ, 222, 165 Cole, A. A., Skillman, E. D., Tolstoy, E., et al. 2007, ApJL, 659, L17 Cole, A. A., Weisz, D. R., Dolphin, A. E., et al. 2014, ApJ, 795, 54 Collins, M. L. M., Chapman, S. C., Rich, R. M., et al. 2013, ApJ, 768, 172 Cooper, M. C., Newman, J. A., Davis, M., Finkbeiner, D. P., \& Gerke, B. F. 2012, spec2d: DEEP2 DEIMOS Spectral Pipeline, Astrophysics Source Code Library, ascl:1203.003

de Vaucouleurs, G., de Vaucouleurs, A., Corwin, H. G., Jr., et al. 1991, in Third Reference Catalogue of Bright Galaxies, ed. G. de Vaucouleurs et al. (New York: Springer)

Dekker, H., Delabre, B., \& Dodorico, S. 1986, Proc. SPIE, 627, 339

Demarque, P., Woo, J.-H., Kim, Y.-C., \& Yi, S. K. 2004, ApJS, 155, 667

Dolphin, A. E., Saha, A., Claver, J., et al. 2002, AJ, 123, 3154

Dolphin, A. E., Saha, A., Skillman, E. D., et al. 2003, AJ, 125, 1261

Faber, S. M., Phillips, A. C., Kibrick, R. I., et al. 2003, Proc. SPIE, 4841, 1657 Fraternali, F., Tolstoy, E., Irwin, M. J., \& Cole, A. A. 2009, A\&A, 499, 121 Frinchaboy, P. M., Majewski, S. R., Muñoz, R. R., et al. 2012, ApJ, 756, 74 Gallazzi, A., Charlot, S., Brinchmann, J., White, S. D. M., \& Tremonti, C. A. 2005, MNRAS, 362, 41

Geha, M., Blanton, M. R., Yan, R., \& Tinker, J. L. 2012, ApJ, 757, 85

Geha, M., Guhathakurta, P., Rich, R. M., \& Cooper, M. C. 2006, AJ, 131, 332

Geha, M., van der Marel, R. P., Guhathakurta, P., et al. 2010, ApJ, 711, 361 Grcevich, J., \& Putman, M. E. 2009, ApJ, 696, 385

Gullieuszik, M., Rejkuba, M., Cioni, M. R., Habing, H. J., \& Held, E. V. 2007, A\&A, 475, 467

Higgs, C. R., McConnachie, A. W., Irwin, M., et al. 2016, MNRAS, 458, 1678

Ho, N., Geha, M., Munoz, R. R., et al. 2012, ApJ, 758, 124

Holtzman, J. A., Afonso, C., \& Dolphin, A. 2006, ApJS, 166, 534

Hunter, D. A., \& Elmegreen, B. G. 2004, AJ, 128, 2170

Hunter, D. A., Elmegreen, B. G., \& Ludka, B. C. 2010, AJ, 139, 447

Hunter, D. A., Ficut-Vicas, D., Ashley, T., et al. 2012, AJ, 144, 134

Karachentsev, I. D., \& Kaisina, E. I. 2013, AJ, 146, 46

Karachentsev, I. D., Kashibadze, O. G., Makarov, D. I., \& Tully, R. B. 2009, MNRAS, 393, 1265

Kirby, E. N., Bullock, J. S., Boylan-Kolchin, M., Kaplinghat, M., \& Cohen, J. G. 2014, MNRAS, 439, 1015

Kirby, E. N., Cohen, J. G., \& Bellazzini, M. 2012, ApJ, 751, 46

Kirby, E. N., Cohen, J. G., Guhathakurta, P., et al. 2013, ApJ, 779, 102

Kirby, E. N., Cohen, J. G., Simon, J. D., \& Guhathakurta, P. 2015a, ApJL, 814, L7

Kirby, E. N., Cohen, J. G., Smith, G. H., et al. 2011a, ApJ, 727, 79

Kirby, E. N., Guhathakurta, P., Bolte, M., Sneden, C., \& Geha, M. C. 2009, ApJ, 705, 328

Kirby, E. N., Guhathakurta, P., Simon, J. D., et al. 2010, ApJS, 191, 352

Kirby, E. N., Guhathakurta, P., \& Sneden, C. 2008, ApJ, 682, 1217

Kirby, E. N., Guo, M., Zhang, A. J., et al. 2015b, ApJ, 801, 125

Kirby, E. N., Lanfranchi, G. A., Simon, J. D., Cohen, J. G., \& Guhathakurta, P. 2011b, ApJ, 727, 78

Kirby, E. N., Simon, J. D., \& Cohen, J. G. 2015c, ApJ, 810, 56

Koch, A., Wilkinson, M. I., Kleyna, J. T., et al. 2009, ApJ, 690, 453

Koleva, M., Prugniel, P., De Rijcke, S., Zeilinger, W. W., \& Michielsen, D. 2009, AN, 330, 960

Koposov, S. E., Gilmore, G., Walker, M. G., et al. 2011, ApJ, 736, 146

Kormendy, J., \& Bender, R. 2012, ApJS, 198, 2

Leaman, R., Venn, K. A., Brooks, A. M., et al. 2012, ApJ, 750, 33

Leaman, R., Venn, K. A., Brooks, A. M., et al. 2013, ApJ, 767, 131

Lee, H., Skillman, E. D., Cannon, J. M., et al. 2006, ApJ, 647, 970

Lee, J. C., Gil de Paz, A., Tremonti, C., et al. 2009, ApJ, 706, 599

Lee, M. G., \& Kim, S. C. 2000, AJ, 119, 777
Li, T. S., Simon, J. D., Drlica-Wagner, A., et al. 2016, ApJ, submitted, arXiv: 1611.05052

Lo, K. Y., Sargent, W. L. W., \& Young, K. 1993, AJ, 106, 507

Lynden-Bell, D. 1975, VA, 19, 299

Mateo, M., Olszewski, E. W., \& Walker, M. G. 2008, ApJ, 675, 201

Mateo, M. L. 1998, ARA\&A, 36, 435

Matteucci, F., \& Greggio, L. 1986, A\&A, 154, 279

Mayer, L., Governato, F., Colpi, M., et al. 2001, ApJ, 559, 754

McConnachie, A. W. 2012, AJ, 144, 4

McConnachie, A. W., Arimoto, N., Irwin, M., \& Tolstoy, E. 2006, MNRAS, 373, 715

Miyazaki, S., Komiyama, Y., Sekiguchi, M., et al. 2002, PASJ, 54, 833

Momany, Y., Clemens, M., Bedin, L. R., et al. 2014, A\&A, 572, A42

Momany, Y., Held, E. V., Saviane, I., et al. 2005, A\&A, 439, 111

Momany, Y., Held, E. V., Saviane, I., \& Rizzi, L. 2002, A\&A, 384, 393

Monelli, M., Gallart, C., Hidalgo, S. L., et al. 2010a, ApJ, 722, 1864

Monelli, M., Hidalgo, S. L., Stetson, P. B., et al. 2010b, ApJ, 720, 1225

Newman, J. A., Cooper, M. C., Davis, M., et al. 2013, ApJS, 208, 5

Orban, C., Gnedin, O. Y., Weisz, D. R., et al. 2008, ApJ, 686, 1030

Ordoñez, A. J., \& Sarajedini, A. 2016, MNRAS, 455, 2163

Pagel, B. E. J. 1997, Nucleosynthesis and Chemical Evolution of Galaxies (1st ed.; Cambridge: Cambridge Univ. Press)

Peñarrubia, J., Navarro, J. F., \& McConnachie, A. W. 2008, ApJ, 673, 226

Revaz, Y., Jablonka, P., Sawala, T., et al. 2009, A\&A, 501, 189

Saha, A., Shaw, R. A., Claver, J. A., \& Dolphin, A. E. 2011, PASP, 123, 481

Saviane, I., Rizzi, L., Held, E. V., Bresolin, F., \& Momany, Y. 2002, A\&A, 390, 59

Schlafly, E. F., \& Finkbeiner, D. P. 2011, ApJ, 737, 103

Schlegel, D. J., Finkbeiner, D. P., \& Davis, M. 1998, ApJ, 500, 525

Schmidt, M. 1963, ApJ, 137, 758

Schroyen, J., de Rijcke, S., Valcke, S., Cloet-Osselaer, A., \& Dejonghe, H. 2011, MNRAS, 416, 601

Schulte-Ladbeck, R. E., Hopp, U., Drozdovsky, I. O., Greggio, L., \& Crone, M. M. 2002, AJ, 124, 896

Searle, L., \& Sargent, W. L. W. 1972, ApJ, 173, 25

Simon, J. D., \& Geha, M. 2007, ApJ, 670, 313

Skillman, E. D., Hidalgo, S. L., Weisz, D. R., et al. 2014, ApJ, 786, 44

Skillman, E. D., Kennicutt, R. C., \& Hodge, P. W. 1989a, ApJ, 347, 875

Skillman, E. D., \& Kennicutt, R. C., Jr. 1993, ApJ, 411, 655

Skillman, E. D., Terlevich, R., \& Melnick, J. 1989b, MNRAS, 240, 563

Sneden, C., Kraft, R. P., Prosser, C. F., \& Langer, G. E. 1992, AJ, 104, 2121

Sohn, S. T., Majewski, S. R., Muñoz, R. R., et al. 2007, ApJ, 663, 960

Spekkens, K., Urbancic, N., Mason, B. S., Willman, B., \& Aguirre, J. E. 2014, ApJL, 795, L5

Spinrad, H., \& Taylor, B. J. 1971, ApJS, 22, 445

Stonkutè, R., Arimoto, N., Hasegawa, T., et al. 2014, ApJS, 214, 19

Sugiura, N. 1978, Commun. Stat. Theor. Methods, 7, 13

Swan, J., Cole, A. A., Tolstoy, E., \& Irwin, M. J. 2016, MNRAS, 456, 4315

Talbot, R. J., Jr., \& Arnett, W. D. 1971, ApJ, 170, 409

Tammann, G. A., Reindl, B., \& Sandage, A. 2011, A\&A, 531, A134

Teyssier, M., Johnston, K. V., \& Kuhlen, M. 2012, MNRAS, 426, 1808

Tollerud, E. J., Beaton, R. L., Geha, M. C., et al. 2012, ApJ, 752, 45

Tollerud, E. J., Geha, M. C., Vargas, L. C., \& Bullock, J. S. 2013, ApJ, 768, 50

Tolstoy, E., Gallagher, J. S., Cole, A. A., et al. 1998, AJ, 116, 1244

van den Bergh, S. 1994, ApJ, 428, 617

van Zee, L. 2000, AJ, 119, 2757

van Zee, L., Haynes, M. P., \& Salzer, J. J. 1997, AJ, 114, 2479

van Zee, L., Skillman, E. D., \& Haynes, M. P. 2006, ApJ, 637, 269

Vargas, L. C., Geha, M. C., \& Tollerud, E. J. 2014a, ApJ, 790, 73

Vargas, L. C., Gilbert, K. M., Geha, M., et al. 2014b, ApJL, 797, L2

Walker, M. G., Belokurov, V., Evans, N. W., et al. 2009a, ApJL, 694, L144

Walker, M. G., Mateo, M., \& Olszewski, E. W. 2009b, AJ, 137, 3100

Walker, M. G., Mateo, M., Olszewski, E. W., et al. 2006, AJ, 131, 2114

Walker, M. G., Mateo, M., Olszewski, E. W., et al. 2007, ApJL, 667, L53

Walker, M. G., Mateo, M., Olszewski, E. W., et al. 2009c, ApJ, 704, 1274

Warren, S. R., Weisz, D. R., Skillman, E. D., et al. 2011, ApJ, 738, 10

Weisz, D. R., Dolphin, A. E., Skillman, E. D., et al. 2014, ApJ, 789, 147

Weisz, D. R., Zucker, D. B., Dolphin, A. E., et al. 2012, ApJ, 748, 88

Wheeler, C., Pace, A. B., Bullock, J. S., et al. 2015, arXiv:1511.01095

Wolf, J., Martinez, G. D., Bullock, J. S., et al. 2010, MNRAS, 406, 1220

Woo, J., Courteau, S., \& Dekel, A. 2008, MNRAS, 390, 1453

Young, L. M., \& Lo, K. Y. 1996, ApJ, 462, 203 